\title{
Longitudinal/Lateral Stability Analysis of Vehicle Motion in the Nonlinear Region
}

\author{
Keji Chen, ${ }^{1}$ Xiaofei Pei, ${ }^{1}$ Guocheng $\mathrm{Ma}^{2}$ and Xuexun $\mathrm{Guo}^{3}$ \\ ${ }^{1}$ Hubei Key Laboratory of Advanced Technology of Automotive Components, Wuhan 430070, China \\ ${ }^{2}$ School of Mechanical \& Engineering, Beijing Institute of Technology, Beijing 100081, China \\ ${ }^{3}$ Hubei Collaborative Innovation Center of Automotive Components Technology, Wuhan 430070, China
}

Correspondence should be addressed to Xiaofei Pei; peixiaofei7@whut.edu.cn

Received 5 April 2016; Revised 16 June 2016; Accepted 10 July 2016

Academic Editor: Yan-Jun Liu

Copyright (C) 2016 Keji Chen et al. This is an open access article distributed under the Creative Commons Attribution License, which permits unrestricted use, distribution, and reproduction in any medium, provided the original work is properly cited.

\begin{abstract}
We focus on the study of motion stability of vehicle nonlinear dynamics. The dynamic model combining with Burckhardt tire model is firstly derived. By phase portrait method, the vehicle stability differences of three cases, front wheels steering/four-wheel steering case, front/rear/four-wheel braking case, and high/low road friction case, are characterized. With the Jacobian matrix, the stable equilibrium point is found and stable areas are calculated out. Similarly, the stability boundaries corresponding to different working conditions are also captured. With vehicle braking or accelerating in the steering process, the relationship between front/rear wheel slippage and the stable area is examined. Comparing with current literatures, the research method and its results present the novelty and provide a guideline for new vehicle controller design.
\end{abstract}

\section{Introduction}

Towards the developing trend of intelligent vehicle, ADAS (advanced driver assistant system) and active safety technology have drawn attention in recent years. Like Ford motor, it proposed a vehicle handling limit warning system based on VSC (vehicle stability control) for the next generation of accident-free vehicle [1]. Currently, the intelligent control system has been designed to handle transient unstable vehicle motion in a guaranteed-safety range. However, the study on the vehicle handling performance, as an essential aspect in vehicle nonlinear dynamics, is never out of date, and a deep insight into the dynamics will bring benefits for advanced controller design.

Facing the challenge of nonlinear system with uncertain dynamic, many intelligent control algorithms such as adaptive fuzzy control and dynamic programming approach are introduced [2-8]. Fuzzy adaptive optimal control was adopted for nonlinear discrete-time systems with dead-zone in [5] and fuzzy adaptive inverse compensation method was used for tracking control of unknown nonlinear system in [6]. Furthermore, [7] outlined a fuzzy adaptive cruise control system which could dynamically adjust the speed of vehicle in accordance with the speed limit of the road. For trajectory tracking problem of mobile robot, [8] presented a model predictive control incorporating neural-dynamic optimization. Instead of optimizing the control algorithm, we focus on the fundamental of vehicle motion stability for controller designing.

With nonlinear properties of the coupled tire forces taken into account, vehicle lateral stability would be affected when longitudinal dynamics is under control. Especially during emergencies, steering behavior with hard brake easily leads the vehicle to drift. It is also known that increasing tire slip ratio reduces the stable region of the vehicle dynamics. Most existing lateral controllers such as VSC and AFS (active front steering) is based on the assumption that wheel slip angle is less than $4 \mathrm{deg}$ [9-11]. For these controllers, only linear properties of tire lateral forces are taken into account. And for longitudinal slip controllers such as ABS and TCS, tire force 
is also restricted in the unsaturated zone. As the tire dynamics is not beyond linear characteristics, ultimate capability of vehicle on slippery road or at high speed is hard to reach. Nowadays, the ICC (integrated chassis control) system has become a research focus because ICC could enable VSC, AFS, and other chassis controllers to work cooperatively to cope with tire nonlinearities [12-14].

In the design of linear controller, the linearized model could be used to represent nonlinear system in the neighborhood of equilibria. Nonlinear vehicle system generally has more than one equilibrium point. Besides, vehicle dynamics are often parameter-dependent. Therefore, a comprehensive understanding of motion stability will provide the knowledge for advanced vehicle dynamic controllers. Phase portrait is a useful method for analysis of vehicle stable region. Reference [15] was the first to propose phase portrait method to describe the variation of sideslip angle and yaw rate in critical motion situation. Thereafter, a saddle-node bifurcation was found with phase portrait method [16]. It was shown that vehicle steering system has one stable and two unstable equilibria. Reference [17] reported bifurcation phenomenon under purely braking maneuvers. Furthermore, a hopf bifurcation was discovered when 4WS (four-wheel steering) is applied in typical road conditions [18]. But this analytical method is complicated for real time controller. Reference [19] introduced a geometric method. The vehicle handling characteristic and equilibria for sharply cornering maneuver is given by joint-point locus geometry. In addition, the lateral stability analyses in [20-22] indicated that the stable equilibrium point disappears when the vehicle speed and steering angle increase over a certain limit. However, the work in [18-22] assumed the constant velocity and zero tire slip ratio, and the effect of the tire slip ratio on lateral vehicle stability was ignored. Therefore, we provide a novel stability analysis for transient vehicle motion on various running conditions by considering the coupling between longitudinal and lateral tire force. The result can be applied to active control design such as the next generation of VSC or ADAS systems. It will help the controller find the stable region of vehicle motion, especially in critical situations. Furthermore, with the guideline the vehicle can conduct some aggressive maneuvers that are unstable seemingly but safe actually.

The estimation of tire/road friction forces is an important aspect in vehicle dynamic studies. References [23-25] proposed many dynamic-based tire models. Since Burckhardt tire model could describe the characteristic of coupled tire force with simple structure [26], its exponential form is more suitable for the large amount of calculation in this paper. If a more precise tire model is used here, the conclusion will be consistent with our analysis but with a more complex procedure and expression.

This paper is organized as follows: a vehicle dynamic model and tire model are firstly presented in Section 2, followed by a primary stability analysis for vehicle lateral motion and coupled longitudinal/lateral motion in Section 3. We then discuss vehicle stable region on various tire slip conditions in Section 4 before concluding the paper in Section 5 .

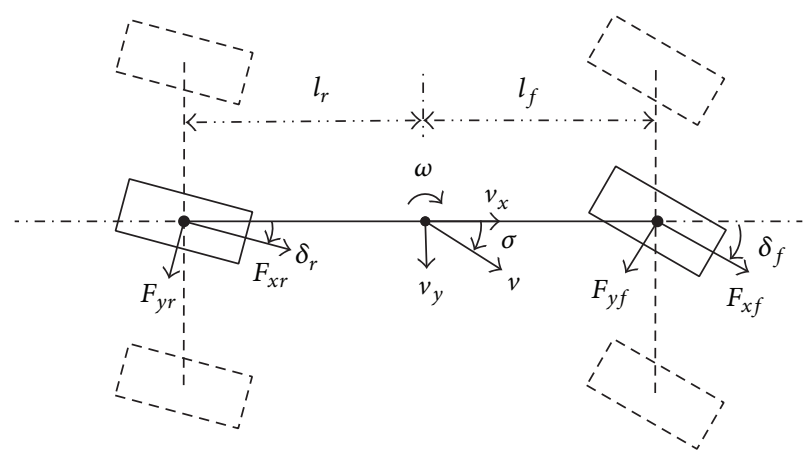

FIgURE 1: Vehicle dynamic model.

\section{Vehicle Dynamics Models}

Any analysis for vehicle motion is based on dynamic model. Here, a classic bicycle model is used for 4WS vehicle, as shown in Figure 1, with the following assumptions:

(1) The origin of $x y$-coordination is fixed at the vehicle's mass center.

(2) Only vehicle planar motion is considered and roll, pitch, vertical motion are ignored.

(3) Steering angles applied to left and right wheels are treated to be equal.

(4) Front track is assumed to be the same as rear track.

In bicycle model, front and rear wheel, respectively, represent the resultant force of left and right wheels. Besides, an additional torque $M_{\text {eq }}$ is applied in yaw moment that results from the difference of the tire forces. Ignoring the air drag and tire rolling resistance, the vehicle dynamic equations,including longitudinal, lateral, and yaw motions, are given in (1) to (3):

$$
\begin{aligned}
m\left(\dot{v}_{x}-v_{y} \omega\right)= & F_{x f} \cos \delta_{f}-F_{y f} \sin \delta_{f}+F_{x r} \cos \delta_{r} \\
& -F_{y r} \sin \delta_{r}, \\
m\left(\dot{v}_{y}+v_{x} \omega\right)= & F_{x f} \sin \delta_{f}+F_{y f} \cos \delta_{f}+F_{x r} \sin \delta_{r} \\
& +F_{y r} \cos \delta_{r}, \\
I_{z} \dot{\omega}= & F_{x f} \sin \delta_{f} l_{f}+F_{y f} \cos \delta_{f} l_{f} \\
& -\left(F_{x r} \sin \delta_{r} l_{r}+F_{y r} \cos \delta_{r} l_{r}\right) \\
& +M_{\mathrm{eq}},
\end{aligned}
$$

where $m$ is vehicle mass; $v_{x}$ is longitudinal velocity; $v_{y}$ is lateral velocity; $\omega$ is vehicle yaw rate; $F_{x f}$ and $F_{x r}$, respectively, represent longitudinal forces of the front and rear tire; $F_{y f}$ and $F_{y r}$, respectively, represent tire lateral forces; $\delta_{f}$ and $\delta_{r}$, respectively, represent wheels steering angles; $I_{z}$ is the yaw moment of vehicle inertia; $l_{f}$ and $l_{r}$ are the distances from vehicle mass center to the front and rear axles. 
TABLE 1: Burckhardt model values.

\begin{tabular}{lccccccccc}
\hline Friction & $k_{1}$ & $k_{2}$ & $k_{3}$ & $k_{x 1}$ & $k_{x 2}$ & $k_{x 3}$ & $k_{y 1}$ & $k_{y 2}$ & $k_{y 3}$ \\
\hline High-mu & -0.726 & 0.3235 & 4.625 & 0.884 & -26.12 & -0.131 & 0.855 & -24.04 & -0.155 \\
Low-mu & -0.716 & 3.642 & 19.889 & 0.185 & -25.5 & -0.052 & 0.193 & -22.4 & -0.046 \\
\hline
\end{tabular}

As Burckhardt tire model is used in the paper, the tire force can be given in

$$
\begin{aligned}
& F_{x}(s, \alpha)=F_{z}\left(k_{1}|\tan (\alpha)|+1\right) \\
& \cdot\left[\operatorname{sgn}(s) k_{x 1}\left(1-e^{\operatorname{sgn}(s) k_{x 2} \cdot s}\right)+k_{x 3} \cdot s\right] \\
& F_{y}(s, \alpha)=-F_{z}\left(\frac{k_{2}|s|+1}{k_{3}|s|+1}\right) \\
& \cdot\left[\operatorname{sgn}(\tan (\alpha)) k_{y 1}\left(1-e^{\operatorname{sgn}(\tan (\alpha)) k_{y 2} \cdot \tan (\alpha)}\right)+k_{y 3}\right. \\
& \cdot \tan (\alpha)]
\end{aligned}
$$

where $F_{x}, F_{y}$, and $F_{z}$ denote longitudinal force, lateral force, and normal force, respectively; $s$ is longitudinal slip ratio; $\alpha$ is sideslip angle; $\operatorname{sgn}(\cdot)$ is the sign function; $k_{1}, k_{2}, k_{3}, k_{x 1}, k_{x 2}$, $k_{x 3}, k_{y 1}, k_{y 2}$, and $k_{y 3}$ are all model parameters, and the values are given for high/low-mu road surface in Table 1.

The front and rear tire/road friction coefficients are defined as follows:

$$
\begin{aligned}
\mu_{f, r} & =\left(k_{1}\left|\tan \left(\alpha_{f, r}\right)\right|+1\right) \\
\cdot & {\left[\operatorname{sgn}\left(s_{f, r}\right) k_{x 1}\left(1-e^{\operatorname{sgn}\left(s_{f, r}\right) k_{x 2} \cdot s_{f, r}}\right)+k_{x 3} \cdot s_{f, r}\right], } \\
\eta_{f, r} & =-\left(\frac{k_{2}\left|s_{f, r}\right|+1}{k_{3}\left|s_{f, r}\right|+1}\right) \\
\cdot & {\left[\operatorname{sgn}\left(\tan \left(\alpha_{f, r}\right)\right) k_{y 1}\left(1-e^{\operatorname{sgn}\left(\tan \left(\alpha_{f, r}\right)\right) k_{y 2} \cdot \tan \left(\alpha_{f, r}\right)}\right)\right.} \\
+ & \left.k_{y 3} \cdot \tan \left(\alpha_{f, r}\right)\right] .
\end{aligned}
$$

Therefore, $F_{x f, r}$ and $F_{y f, r}$ can be expressed as

$$
\begin{aligned}
& F_{x f, r}=F_{z f, r} \cdot \mu_{f, r}, \\
& F_{y f, r}=F_{z f, r} \cdot \eta_{f, r} .
\end{aligned}
$$

When vehicle brakes hard, the longitudinal load transfer cannot be neglected. The loads of front and rear tire could be given by

$$
\begin{aligned}
F_{z f} & =\frac{l_{r}}{l_{f}+l_{r}} m g-\frac{h}{l_{f}+l_{r}}\left(F_{z f} \mu_{f} \cos \delta_{f}\right. \\
& \left.-F_{z f} \eta_{f} \sin \delta_{f}+F_{z r} \mu_{r} \cos \delta_{r}-F_{z r} \eta_{r} \sin \delta_{r}\right), \\
F_{z r} & =\frac{l_{f}}{l_{f}+l_{r}} m g-\frac{h}{l_{f}+l_{r}}\left(F_{z f} \mu_{f} \cos \delta_{f}-F_{z f} \eta_{f} \sin \delta_{f}\right. \\
& \left.+F_{z r} \mu_{r} \cos \delta_{r}-F_{z r} \eta_{r} \sin \delta_{r}\right),
\end{aligned}
$$

where $h$ is the height of vehicle mass center.
By transformation, the load transfer coefficients are defined as

$$
\begin{gathered}
\zeta_{f}=\frac{l_{r}-P_{r}}{L+P_{f}-P_{r}}, \\
\zeta_{r}=\frac{l_{f}+P_{f}}{L+P_{f}-P_{r}},
\end{gathered}
$$

where $P_{f}=\left(u_{f} \cos \delta_{f}-\eta_{f} \sin \delta_{f}\right) h ; P_{r}=\left(u_{r} \cos \delta_{r}-\right.$ $\left.\eta_{r} \sin \delta_{r}\right) h$.

Therefore, $F_{z f, r}$ can be given in

$$
F_{z f, r}=m g \zeta_{f, r} .
$$

Due to differences of left and right tire forces, the extra torque $M_{\mathrm{eq}}$ can be expressed as

$$
\begin{aligned}
M_{\mathrm{eq}}= & \Delta F_{z f} \eta_{f} B \sin \delta_{f}-\Delta F_{z f} \mu_{f} B \cos \delta_{f} \\
& +\Delta F_{z r} \eta_{r} B \sin \delta_{r}-\Delta F_{z r} \mu_{r} B \cos \delta_{r},
\end{aligned}
$$

where $B$ is the half wheel track; $\Delta F_{z f}$ and $\Delta F_{z r}$ are the normal force differences between left and right tire, which can be expressed as follows:

$$
\begin{aligned}
& \Delta F_{z f, r}=\Delta F_{z} \zeta_{f, r}=-\frac{h}{B}\left(F_{x f} \sin \delta_{f}+F_{y f} \cos \delta_{f}\right. \\
& \left.\quad+F_{x r} \sin \delta_{r}+F_{y r} \cos \delta_{r}\right) \zeta_{f, r} .
\end{aligned}
$$

Let $\sigma$ denote the tangent value of vehicle sideslip angle; namely, $\sigma=v_{y} / v_{x}$. Then the sideslip angles of front and rear tire can be expressed as follows:

$$
\begin{gathered}
\alpha_{f}=\arctan \left(\sigma+\frac{l_{f} \omega}{v_{x}}\right)-\delta_{f}, \\
\alpha_{r}=\arctan \left(\sigma-\frac{l_{r} \omega}{v_{x}}\right)-\delta_{r} .
\end{gathered}
$$

Besides, the lateral velocity in (2) can be derived according to kinematic relations:

$$
\dot{v}_{y}=\dot{\sigma} v_{x}+\dot{v}_{x} \sigma
$$

Then taking $\sigma$ and $\omega$ as state variables, the governing equations for vehicle planar motion at a certain instantaneous $v_{x}, \delta_{f, r}$, and $s_{f, r}$ could be expressed as follows:

$$
\begin{aligned}
\dot{\sigma} & =\frac{g}{v_{x}}\left(\zeta_{f} Q_{f}+\frac{\sigma}{h} \zeta_{f} P_{f}+\zeta_{r} Q_{r}+\frac{\sigma}{h} \zeta_{r} P_{r}\right)-\omega\left(\sigma^{2}\right. \\
& +1) \\
\dot{\omega} & =\frac{m g}{I_{z}}\left[\left(l_{f} \zeta_{f} Q_{f}-l_{r} \zeta_{r} Q_{r}\right)\right. \\
& \left.+\left(\zeta_{f} P_{f}+\zeta_{r} P_{r}\right)\left(\zeta_{f} Q_{f}+\zeta_{r} Q_{r}\right)\right]
\end{aligned}
$$


TABLE 2: Vehicle model values.

\begin{tabular}{lccccc}
\hline$m(\mathrm{~kg})$ & $I_{z}\left(\mathrm{~kg} \cdot \mathrm{m}^{2}\right)$ & $h(\mathrm{~m})$ & $l_{f}(\mathrm{~m})$ & $l_{r}(\mathrm{~m})$ & $B(\mathrm{~m})$ \\
\hline 1295 & 1580 & 0.469 & 0.946 & 1.526 & 0.715 \\
\hline
\end{tabular}

where $Q_{f}=\mu_{f} \sin \delta_{f}+\eta_{f} \cos \delta_{f} ; Q_{r}=\mu_{r} \sin \delta_{r}+\eta_{r} \cos \delta_{r}$. And all related parameters values are shown in Table 2.

\section{Vehicle Stability Characteristic Analysis by Phase Portrait}

In this section, vehicle stability analysis is discussed by using phase portrait method. The phase portraits are obtained by numerically solving (14) and (15) with Runge-Kutta method under series of initial states of vehicle motion. In all the phase portraits, " $x$ " denotes an initial vehicle state and the corresponding curve denotes the locus of the vehicle motion states. Additionally, we define attraction domain of the nonlinear dynamical systems as the stable region at the given vehicle motion and input. The vehicle motion is considered to be stable when vehicle motion states are located within the stable region.

3.1. Stability Analysis for Pure Steering. In this part, we extend the lateral stability analyses in [18-22]. More comprehensive operating conditions (e.g., high/low friction road and 4WS control) are taken into account. We use the phase portrait method to analyze vehicle motion stability under steering action. The result also provides a foundation for further comparison with braking and steering in latter part.

Figure 2 illustrates $\omega$ - $\sigma$ dynamical characteristics with steering angle $\delta_{f}=0.02 \mathrm{rad}$ and $\delta_{r}=0 \mathrm{rad}$, respectively. We observe that the stable region becomes narrower with increasing velocity $v_{x}$ when the vehicle corners. Figures 2 (b) and 2 (d) show that the vehicle fails to maintain a steady state at $35 \mathrm{~m} / \mathrm{s}$ on high-mu road surface, or $15 \mathrm{~m} / \mathrm{s}$ on low friction road, as there is no stable region in the phase portraits. Therefore, the front wheels steering maneuver is forbidden when vehicle exceeds a critical speed.

Figure 3 shows the $\omega-\sigma$ phase portraits under the change of steering angle $\delta_{f}$. We observe the following facts from this plot:

(1) Increasing steering angle $\delta_{f}$ will reduce the stable region.

(2) The stable equilibrium point disappears at a critical steering angle $\delta_{f}$, such as 0.08 rad in Figure 3(c) and $0.02 \mathrm{rad}$ in Figure 3(f).

(3) The stable equilibrium point is at the origin when $\delta_{f}=0 \mathrm{rad}$. For nonzero steering angle, the stable equilibrium point is no longer fixed at the origin.

It can be summarized from Figures 2 and 3 that whether velocity $v_{x}$ or steering angle $\delta_{f}$ increases, the stable equilibrium point will move to the unstable saddle point. Furthermore, the stable equilibrium point will merge with one of the unstable saddle equilibria at certain condition. Then there is no stable region.
Besides, the $\omega-\sigma$ phase portraits are compared between high-mu friction and low-mu friction conditions. For highmu road surface, the stable region is much larger and is more robust to the change of the vehicle velocity and steering angle. While for low-mu road surface, a slight steering maneuver could easily make the vehicle unstable (see Figures 3(d), 3(e), and 3(f)).

A similar stability analysis is given for $4 \mathrm{WS}$ vehicle in Figures 4 and 5. The rear wheels steering is taken into account to compensate the oversteer of the front wheels. Here, a compliance steering strategy is adopted for 4WS control, which is mathematically expressed as

$$
\delta_{r}=K \delta_{f}
$$

where $K$ is a parameter that represents the linear relationship between $\delta_{f}$ and $\delta_{r}$.

Without losing generality, $K$ is set to 0.5 in Figures 4 and 5 . Although the two phase portraits look similar to those shown in Figures 2 and 3, we can observe that the risk of instability is reduced by the rear wheels steering. Under the same $v_{x}$ and $\delta_{f}$ conditions, 2WS (front wheels steering) vehicle cannot keep stable motion in Figures 2(b), 2(d), 3(c), and 3(f), but 4WS vehicle is still in safe area as showed by Figures 4(a), 4(b), 5(a), and 5(b).

3.2. Stability Analysis for Steering and Braking. A proper braking maneuver could increase the understeer tendency when vehicle is in high speed cornering, such as the principle of VSC. On the contrary, applying a rude brake will deteriorate the vehicle stability severely. In this part, we analyze the coupled longitudinal and lateral stability under various vehicle operation conditions.

Figures 6 and 7 illustrate the phase portraits of vehicle planar motion for $2 \mathrm{WS}$ and $4 \mathrm{WS}$, respectively. As seen in Figures 6(a), 6(d), 7(a), and 7(d), the stable region expands when applying brake force on front wheels. Similar to 4WS control, front wheels braking can increase the tendency of understeer for 2WS vehicle. Figures 6(b), 6(e), 7(b), and 7(e) show that rear wheels braking totally destruct the formation of stable region even for $4 \mathrm{WS}$ vehicle on high-mu road surface. In other words, the vehicle will lose its stability immediately at any initial condition, if only the rear wheels brake during cornering. This situation is dangerous and should be avoided. When the four-wheel braking is applied, we can find the stability of 2WS vehicle is improved by comparing Figures 6(c) and 6(f) with Figures 3(c) and 3(f), but the stability of $4 \mathrm{WS}$ vehicle worsens comparing Figures 7(c) and 7(f) with Figures 5(b) and 5(d). Therefore, fourwheel braking is only useful for $2 \mathrm{WS}$ vehicle in some cases.

\section{Vehicle Stability Boundary Analysis by Jacobian Matrix}

In this section, we further figure out the stability boundary according to various steering, braking, or road friction conditions. As is analyzed above, the structures of phase portraits change with specific operation conditions. When the stable equilibrium point merges with the unstable saddle 


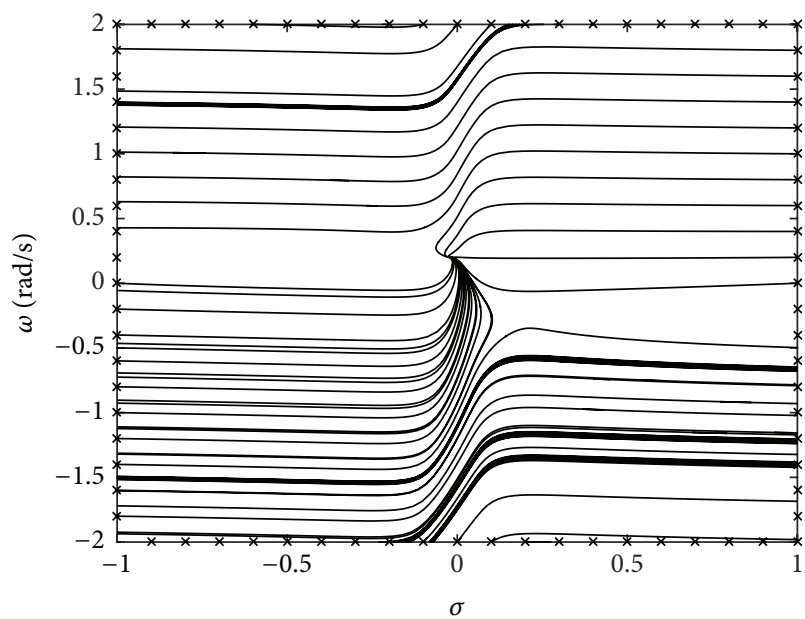

(a) $v_{x}=25 \mathrm{~m} / \mathrm{s}$

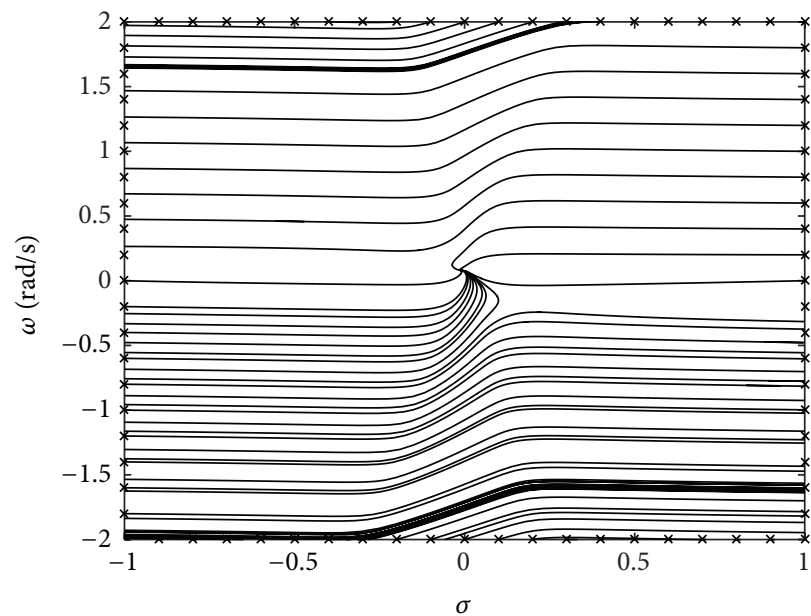

(c) $v_{x}=10 \mathrm{~m} / \mathrm{s}$

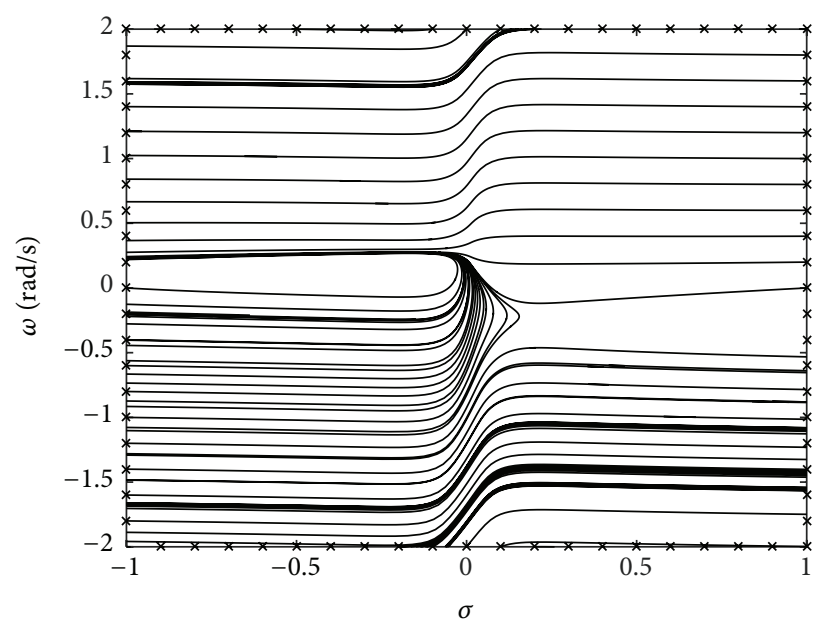

(b) $v_{x}=35 \mathrm{~m} / \mathrm{s}$

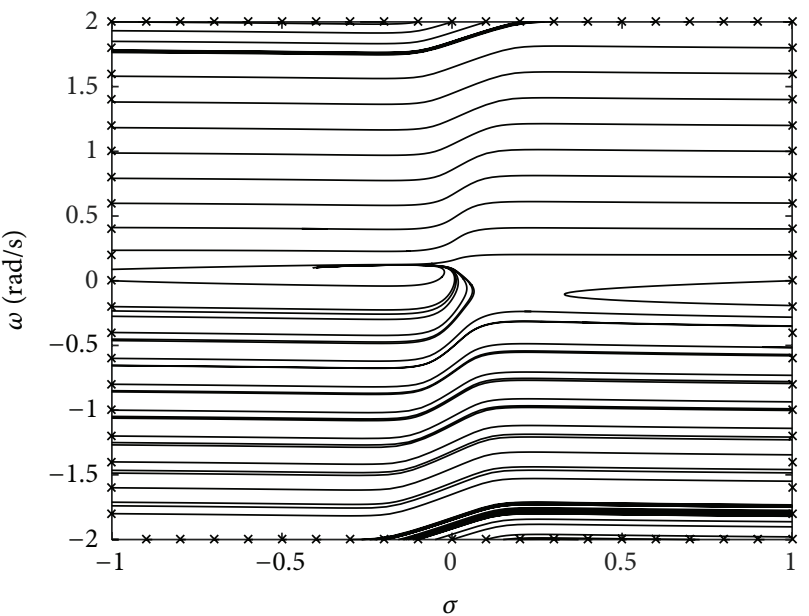

(d) $v_{x}=15 \mathrm{~m} / \mathrm{s}$

FIGURE 2: Phase portraits for $\delta_{f}=0.02 \mathrm{rad}$ and $\delta_{r}=0 \mathrm{rad}$. (a) and (b) On high friction road; (c) and (d) on low friction road.

point, there is no stable region in the phase plane. We assume that if a stable equilibrium point exists, the corresponding vehicle motion is stable. Therefore, we could determine vehicle motion stability by the eigenvalues of the Jacobian matrix at each equilibrium point. Let $f_{1}$ denote the transform of (15) and let $f_{2}$ denote the transform of (16); the Jacobian matrix $\mathbf{J}$ is given as

$$
\mathbf{J}=\frac{\partial \mathbf{f}}{\partial \mathbf{x}}=\left[\begin{array}{ll}
\frac{\partial f_{1}}{\partial x_{1}} & \frac{\partial f_{1}}{\partial x_{2}} \\
\frac{\partial f_{2}}{\partial x_{1}} & \frac{\partial f_{2}}{\partial x_{2}}
\end{array}\right]
$$

where

$$
\begin{array}{r}
\frac{\partial f_{1}}{\partial x_{1}}=\frac{g}{v_{x}}\left[\frac{\partial\left(\zeta_{f} Q_{f}\right)}{\partial \sigma}-\frac{1}{h}\left(\zeta_{f} P_{f}+\sigma \frac{\partial\left(\zeta_{f} P_{f}\right)}{\partial \sigma}\right)\right. \\
\left.+\frac{\partial\left(\zeta_{r} Q_{r}\right)}{\partial \sigma}-\frac{1}{h}\left(\zeta_{r} P_{r}+\sigma \frac{\partial\left(\zeta_{r} P_{r}\right)}{\partial \sigma}\right)\right]-2 \sigma \omega
\end{array}
$$

$$
\begin{aligned}
\frac{\partial f_{1}}{\partial x_{2}} & =\frac{g}{v_{x}}\left(\frac{\partial\left(\zeta_{f} Q_{f}\right)}{\partial \omega}-\frac{\sigma}{h} \frac{\partial\left(\zeta_{f} P_{f}\right)}{\partial \omega}+\frac{\partial\left(\zeta_{r} Q_{r}\right)}{\partial \omega}\right. \\
- & \left.\frac{\sigma}{h} \frac{\partial\left(\zeta_{r} P_{r}\right)}{\partial \omega}\right)-\left(\sigma^{2}+1\right), \\
\frac{\partial f_{2}}{\partial x_{1}} & =\frac{m g}{I_{z}}\left[\left(l_{f} \frac{\partial\left(\zeta_{f} Q_{f}\right)}{\partial \sigma}-l_{r} \frac{\partial\left(\zeta_{r} Q_{r}\right)}{\partial \sigma}\right)\right. \\
+ & \left(\frac{\partial\left(\zeta_{f} P_{f}\right)}{\partial \sigma}+\frac{\partial\left(\zeta_{r} P_{r}\right)}{\partial \sigma}\right)\left(\zeta_{f} Q_{f}+\zeta_{r} Q_{r}\right) \\
+ & \left.\left(\zeta_{f} P_{f}+\zeta_{r} P_{r}\right)\left(\frac{\partial\left(\zeta_{f} Q_{f}\right)}{\partial \sigma}+\frac{\partial\left(\zeta_{r} Q_{r}\right)}{\partial \sigma}\right)\right], \\
\frac{\partial f_{2}}{\partial x_{2}} & =\frac{m g}{I_{z}}\left[\left(l_{f} \frac{\partial\left(\zeta_{f} Q_{f}\right)}{\partial \omega}-l_{r} \frac{\partial\left(\zeta_{r} Q_{r}\right)}{\partial \omega}\right)\right.
\end{aligned}
$$




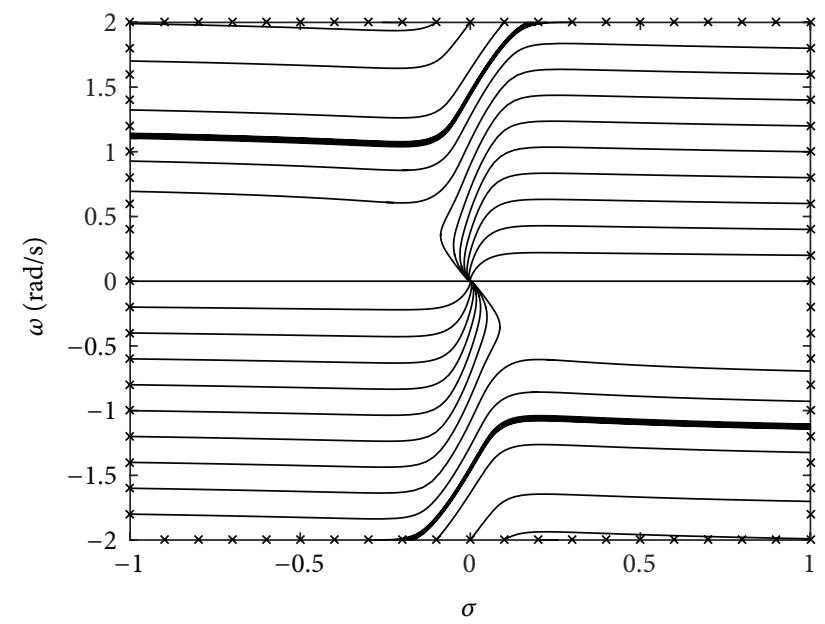

(a) $\delta_{f}=0 \mathrm{rad}$

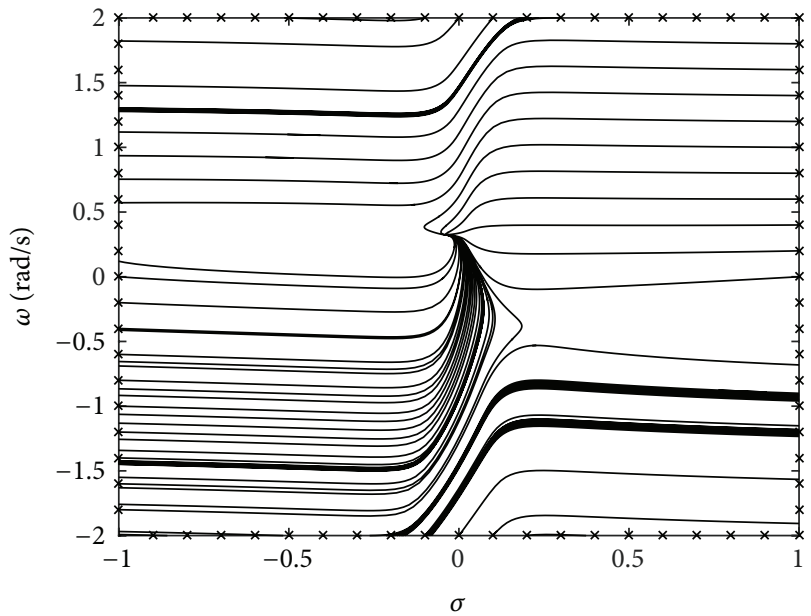

(b) $\delta_{f}=0.04 \mathrm{rad}$

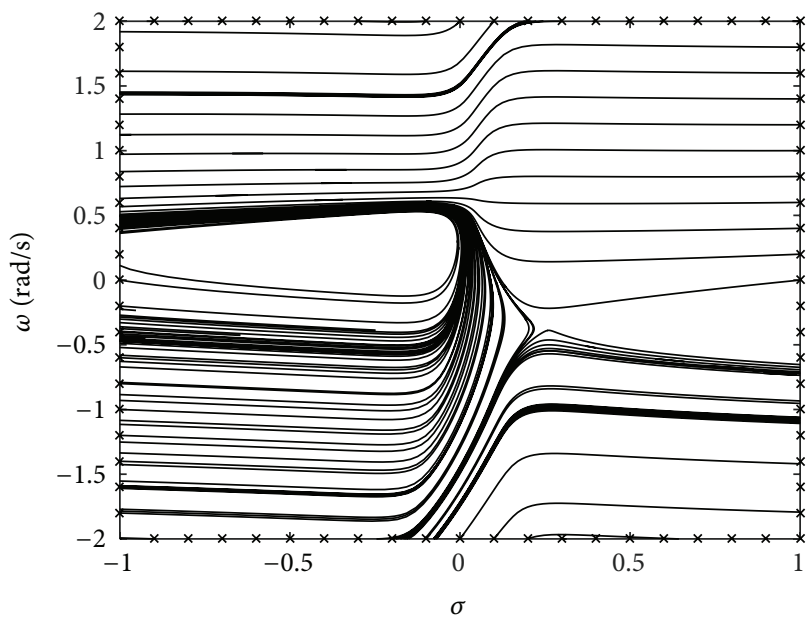

(c) $\delta_{f}=0.08 \mathrm{rad}$

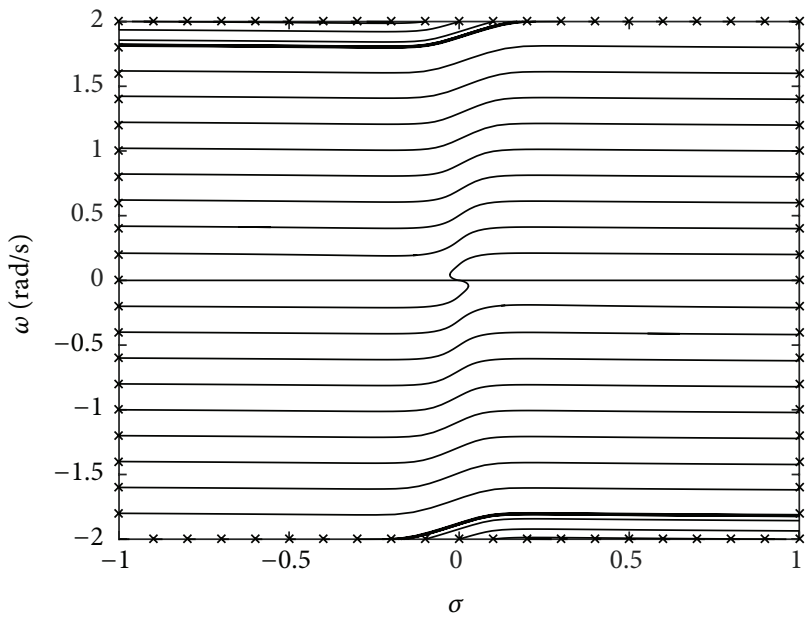

(d) $\delta_{f}=0 \mathrm{rad}$

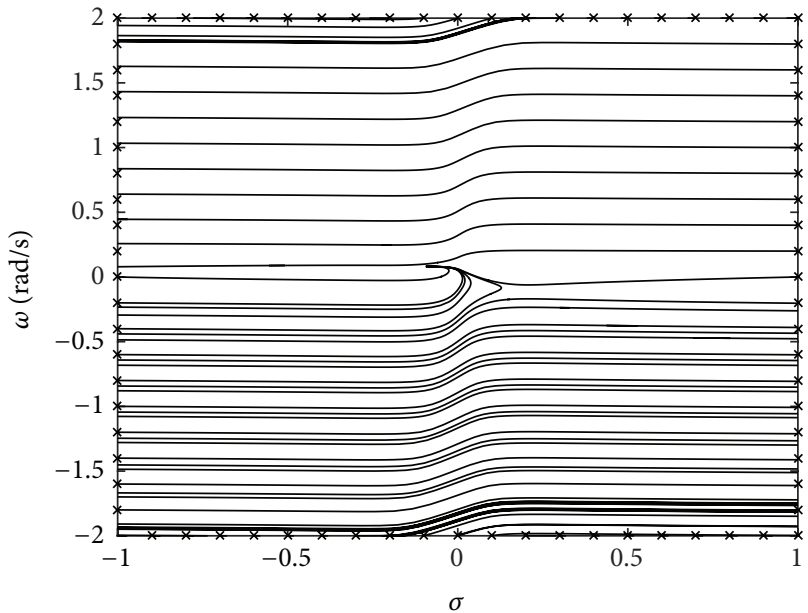

(e) $\delta_{f}=0.01 \mathrm{rad}$

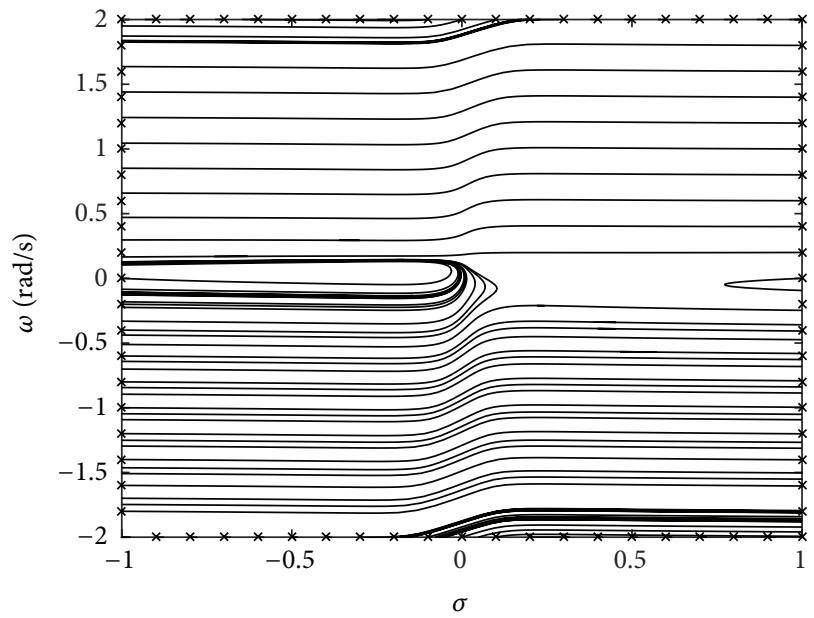

(f) $\delta_{f}=0.02 \mathrm{rad}$

FIGURE 3: Phase portraits for $v_{x}=20 \mathrm{~m} / \mathrm{s}$ and $\delta_{r}=0 \mathrm{rad}$. (a), (b), and (c) On high friction road; (d), (e), and (f) on low friction road. 


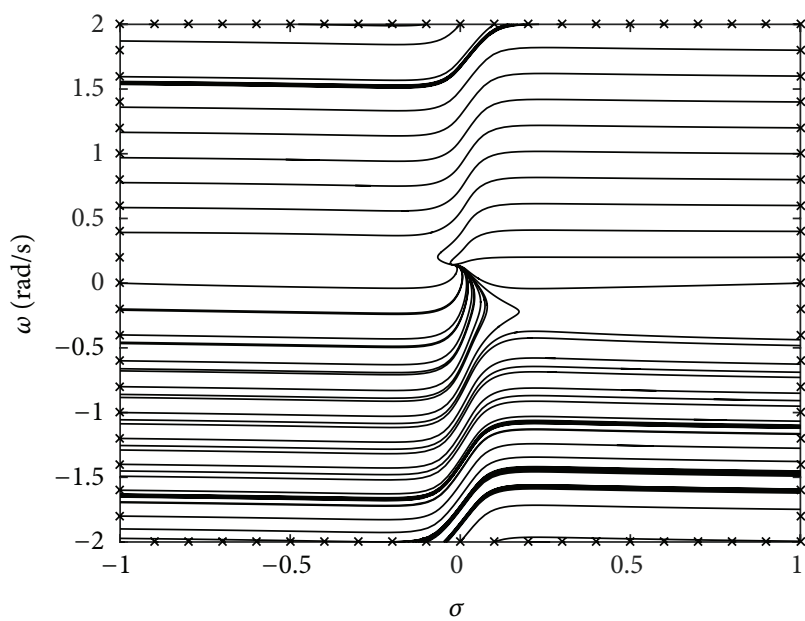

(a) $v_{x}=35 \mathrm{~m} / \mathrm{s}$

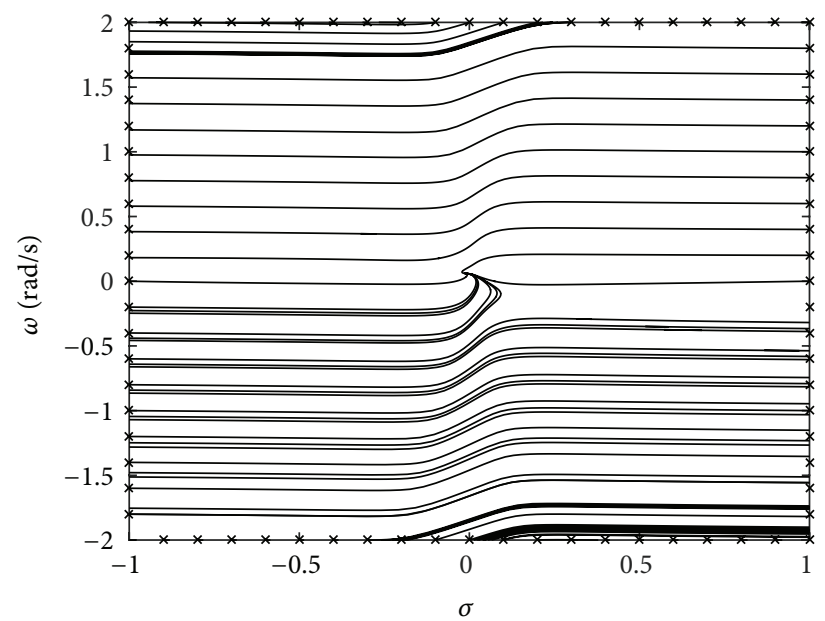

(b) $v_{x}=15 \mathrm{~m} / \mathrm{s}$

FIgURE 4: Phase portraits for $\delta_{f}=0.02 \mathrm{rad}$ and $K=0.5$. (a) On high friction road; (b) on low friction road.

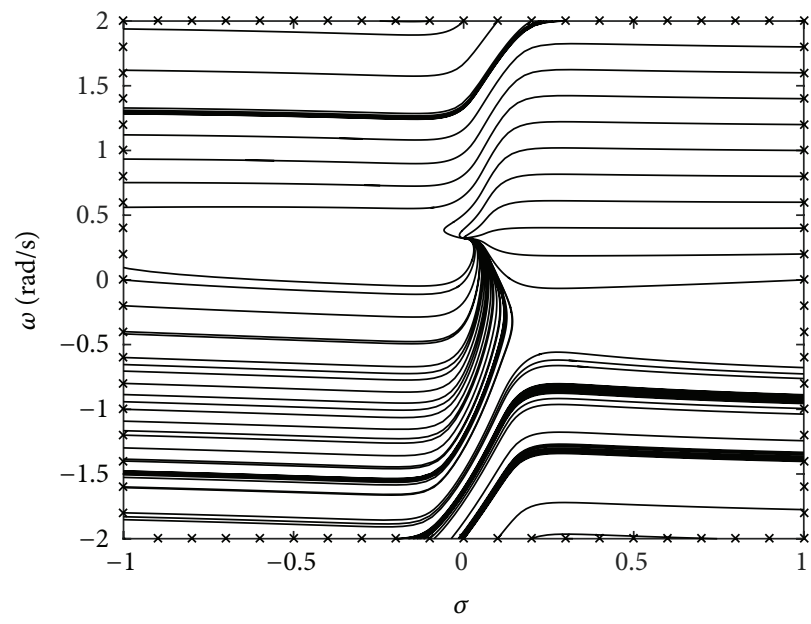

(a) $\delta_{f}=0.08 \mathrm{rad}$

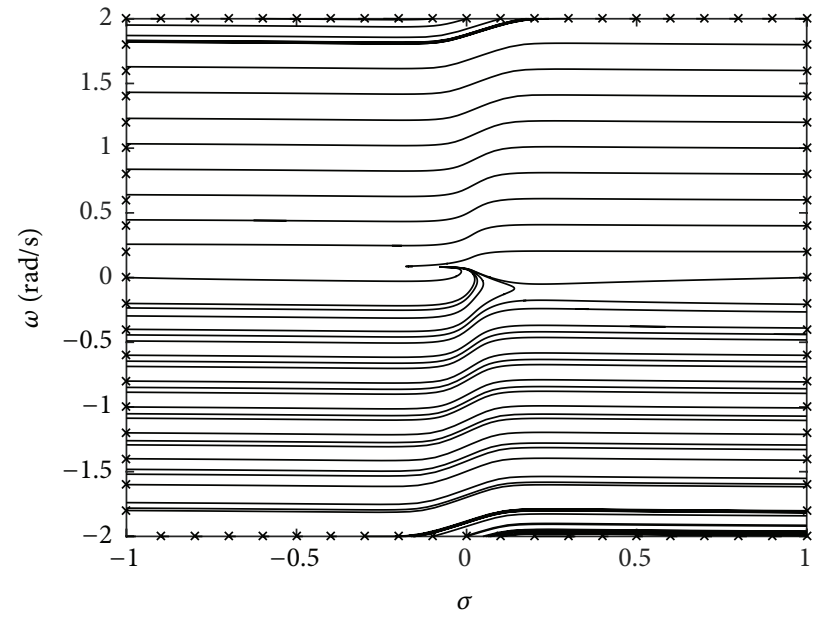

(b) $\delta_{f}=0.02 \mathrm{rad}$

Figure 5: Phase portraits for $v_{x}=20 \mathrm{~m} / \mathrm{s}$ and $K=0.5$. (a) On high friction road; (b) on low friction road.

$$
\begin{aligned}
& +\left(\frac{\partial\left(\zeta_{f} P_{f}\right)}{\partial \omega}+\frac{\partial\left(\zeta_{r} P_{r}\right)}{\partial \omega}\right)\left(\zeta_{f} Q_{f}+\zeta_{r} Q_{r}\right) \\
& \left.+\left(\zeta_{f} P_{f}+\zeta_{r} P_{r}\right)\left(\frac{\partial\left(\zeta_{f} Q_{f}\right)}{\partial \omega}+\frac{\partial\left(\zeta_{r} Q_{r}\right)}{\partial \omega}\right)\right] .
\end{aligned}
$$

The remaining expressions are given in the appendix.

The stability criterion of vehicle motion at the equilibria is defined as follows: if all the eigenvalues of the matrix $\mathbf{J}$ have negative real parts, the equilibrium is considered to be stable under the current condition. If not, no stable equilibrium exists. The stability boundaries divide the area of operating conditions into stability and instability. The " $S$ " area is for the stable equilibrium while " $U$ " area is for the unstable equilibrium. It is easy to obtain the size of stable region from Figures 9-12.
4.1. The Stable Area of Operating Conditions $v_{x}-\delta_{f}$. Firstly, pure steering condition is analyzed. In Figure 8 , the stable area of operating conditions $v_{x}-\delta_{f}$ is narrow at the top and wide at the bottom. It means that the safe range of steering angle becomes narrower as the velocity $v_{x}$ increases. In other words, the safe range of longitudinal velocity shrinks as the steering angle increases. Similar to the result from the phase portraits in the previous section, 4WS vehicle with high friction has the largest stable area, relatively, while $2 \mathrm{WS}$ vehicle with low friction has the smallest stable area under the same $v_{x}$ and $\delta_{f}$ input. In addition, the comparison of Figures $8(\mathrm{a})$ and 8 (b) indicates that the $4 \mathrm{WS}$ system provides a better steering capability when $v_{x}>16 \mathrm{~m} / \mathrm{s}$ on high-mu road surface. However, on low-mu road surface, the effect of 4WS is fairly limited at high speed from Figures $8(\mathrm{c})$ and $8(\mathrm{~d})$.

Then, we take steering and braking operation into account from Figures 9-11. In Figure 9, we discuss the influences of different braking modes on the stable region 


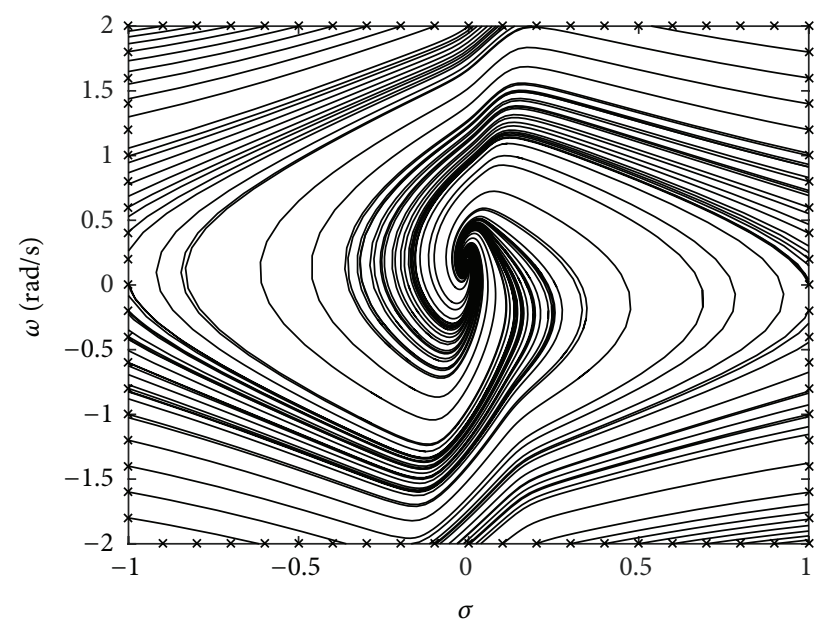

(a) $s_{f}=-0.2, s_{r}=0$

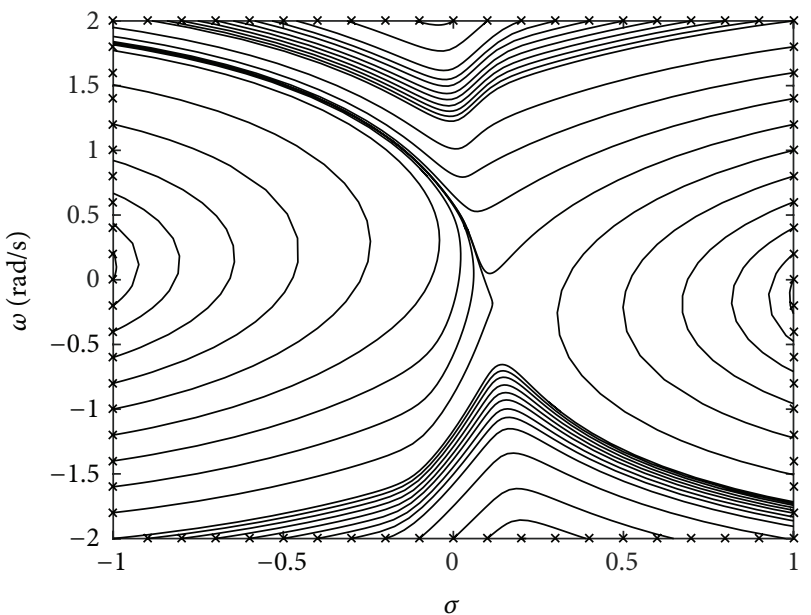

(b) $s_{f}=0, s_{r}=-0.2$

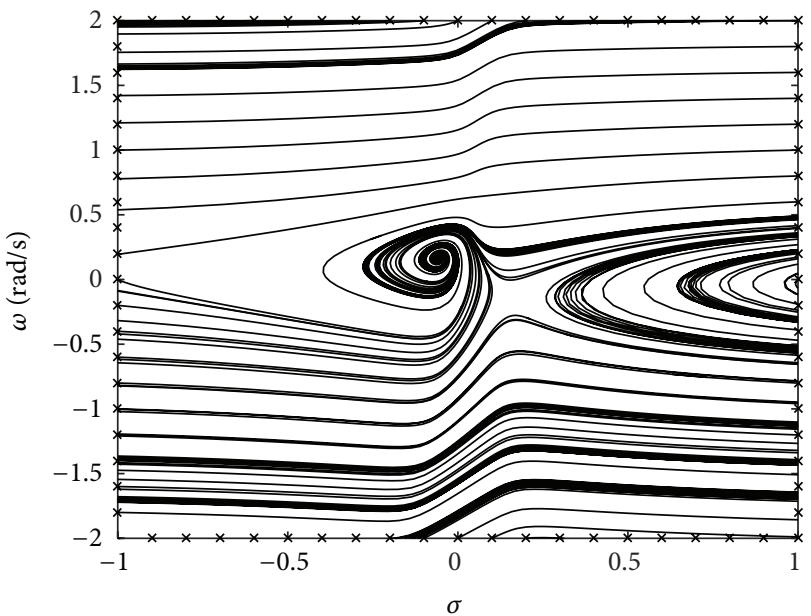

(c) $s_{f}=-0.2, s_{r}=-0.2$

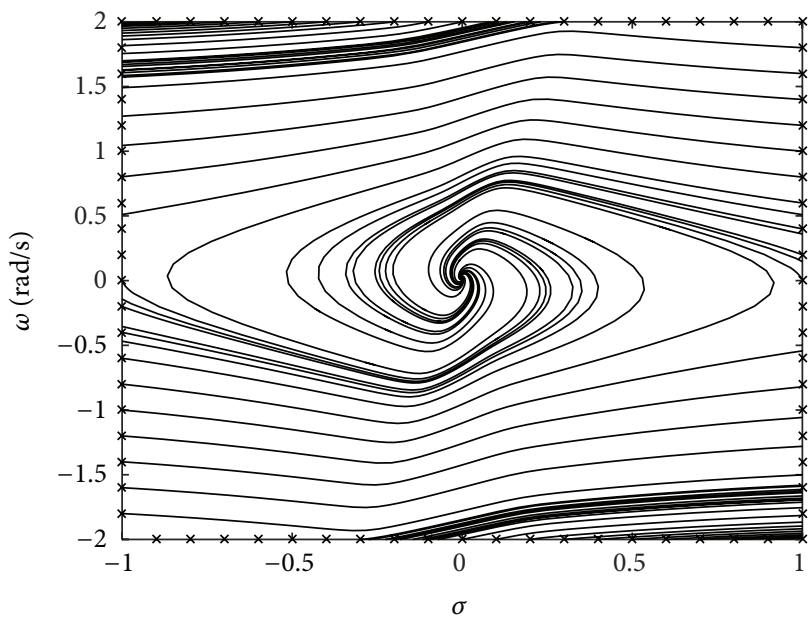

(d) $s_{f}=-0.2, s_{r}=0$

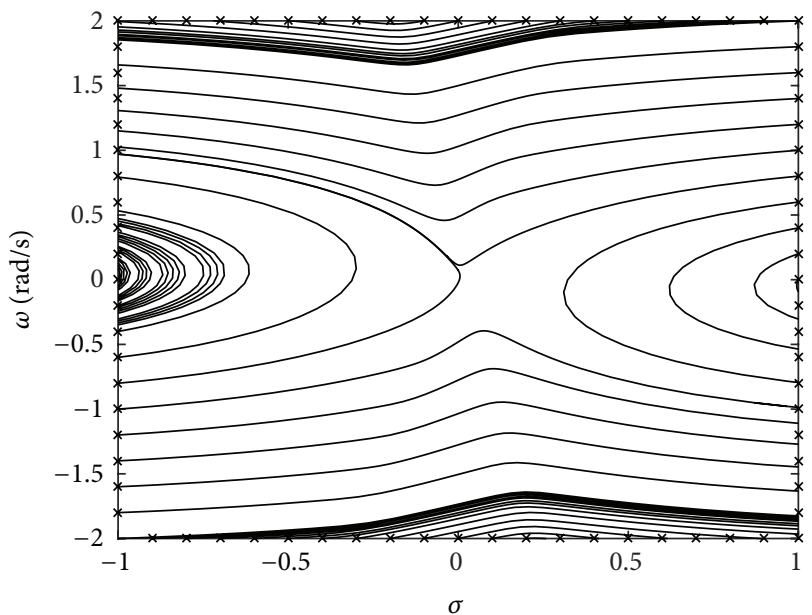

(e) $s_{f}=0, s_{r}=-0.2$

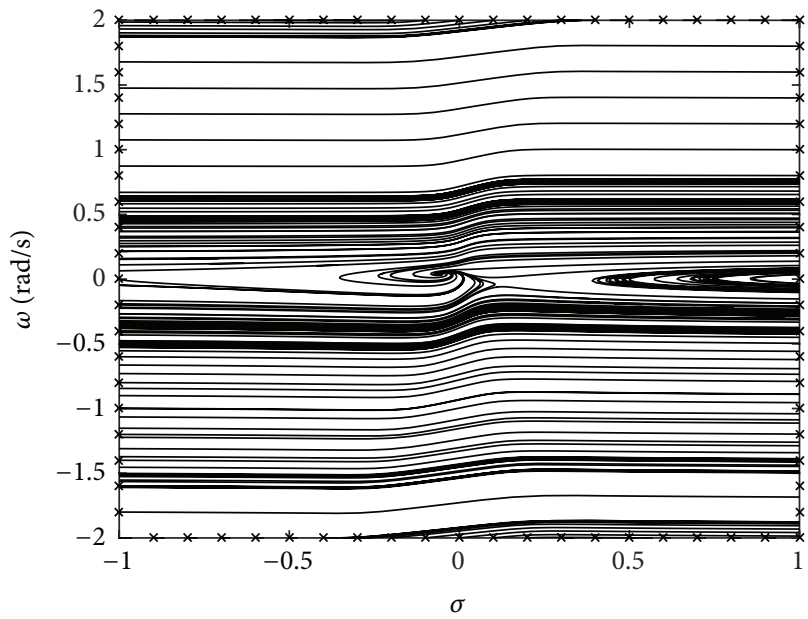

(f) $s_{f}=-0.2, s_{r}=-0.2$

Figure 6: Phase portraits for 2WS vehicle. (a), (b), and (c) $v_{x}=20 \mathrm{~m} / \mathrm{s}$ and $\delta_{f}=0.08 \mathrm{rad}$ on high friction road; (d), (e), and (f) $v_{x}=10 \mathrm{~m} / \mathrm{s}$ and $\delta_{f}=0.02 \mathrm{rad}$ on low friction road. 


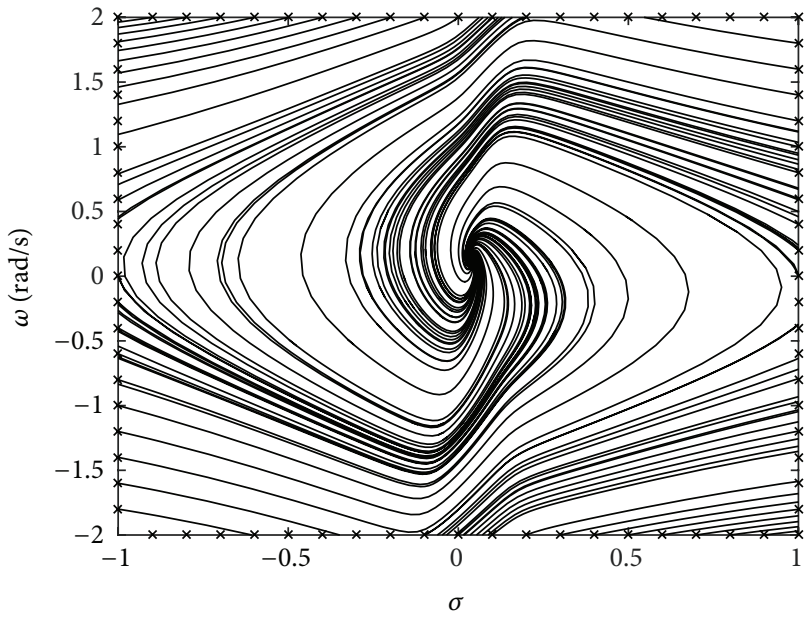

(a) $s_{f}=-0.2, s_{r}=0$

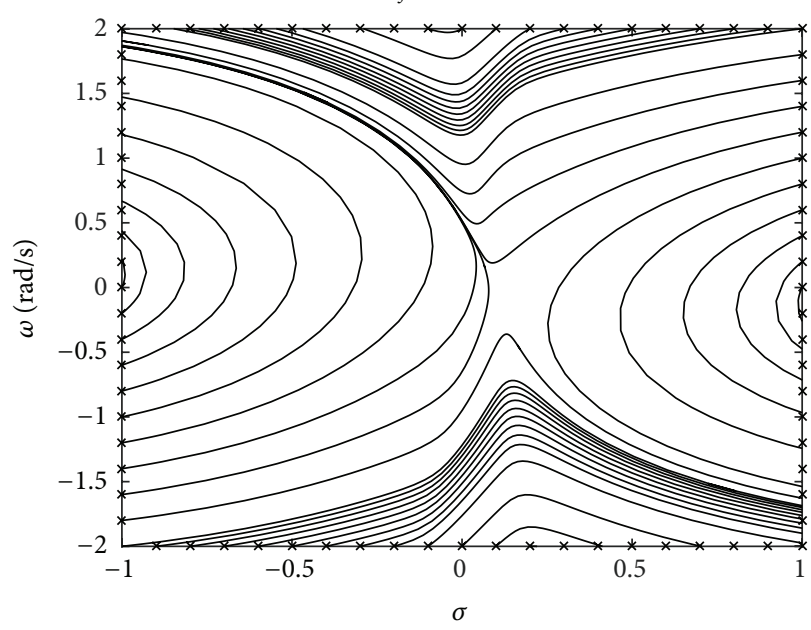

(b) $s_{f}=0, s_{r}=-0.2$

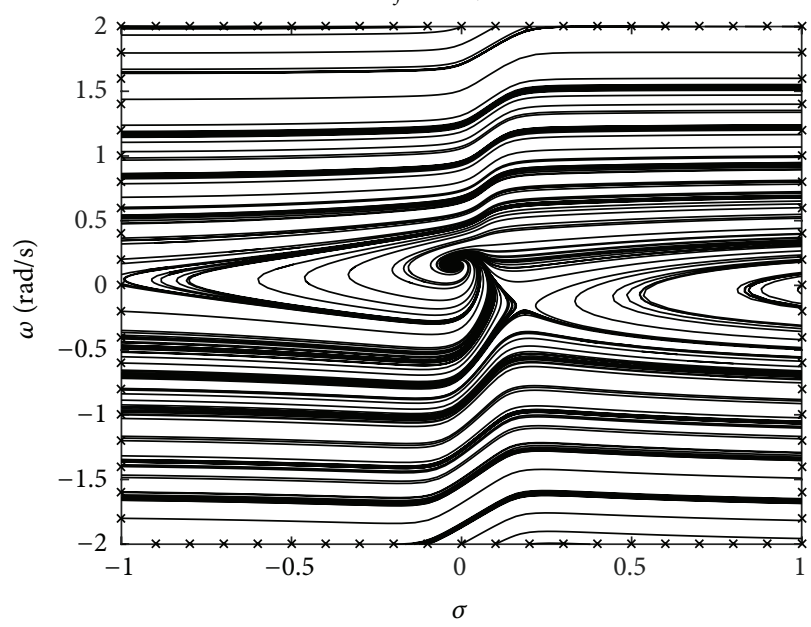

(c) $s_{f}=-0.2, s_{r}=-0.2$

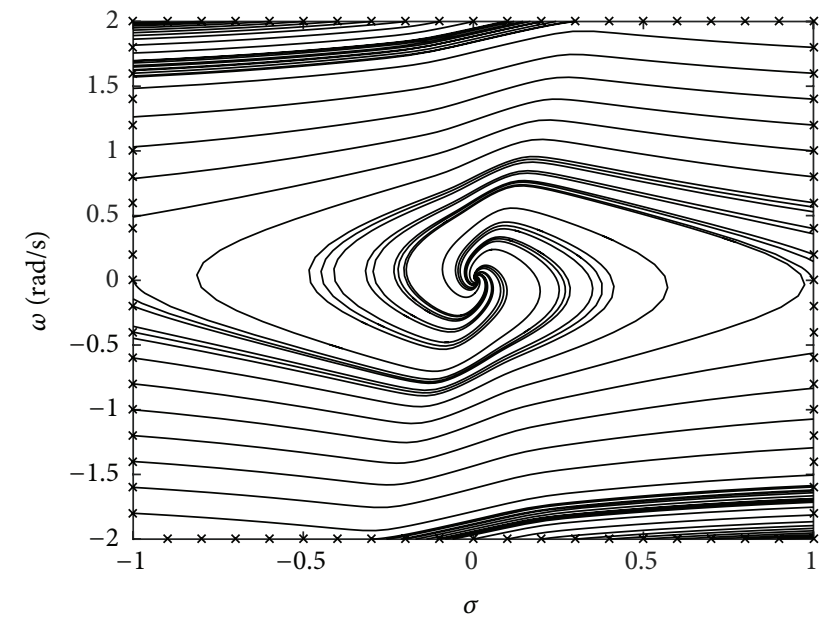

(d) $s_{f}=-0.2, s_{r}=0$

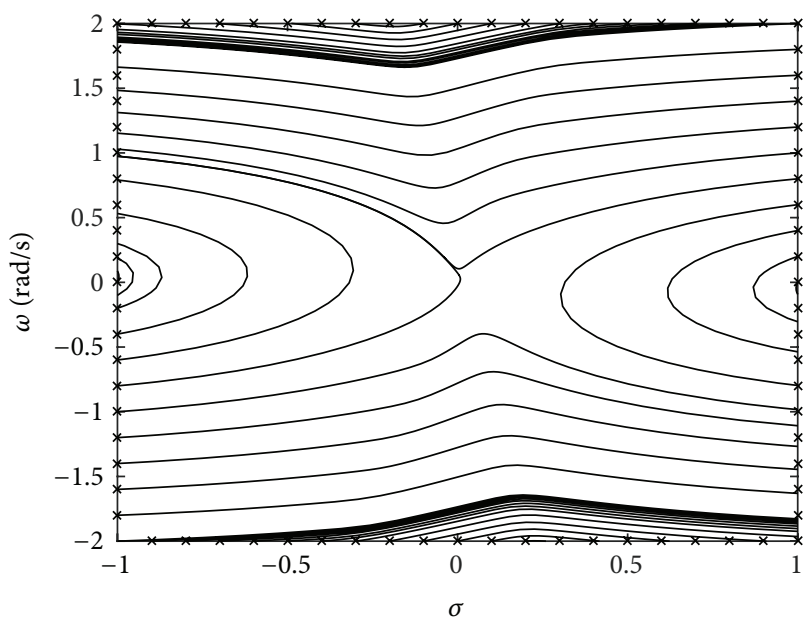

(e) $s_{f}=0, s_{r}=-0.2$

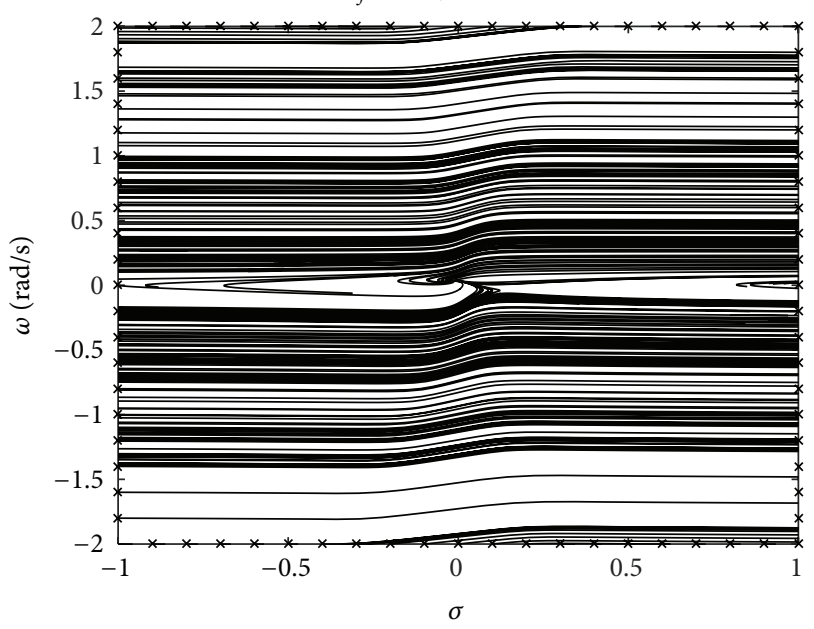

(f) $s_{f}=-0.2, s_{r}=-0.2$

FiguRe 7: Phase portraits for 4WS vehicle with $K=0.5$. (a), (b), and (c) $v_{x}=20 \mathrm{~m} / \mathrm{s}$ and $\delta_{f}=0.08$ rad on high friction road; (d), (e), and (f) $v_{x}=10 \mathrm{~m} / \mathrm{s}$ and $\delta_{f}=0.02 \mathrm{rad}$ on low friction road. 


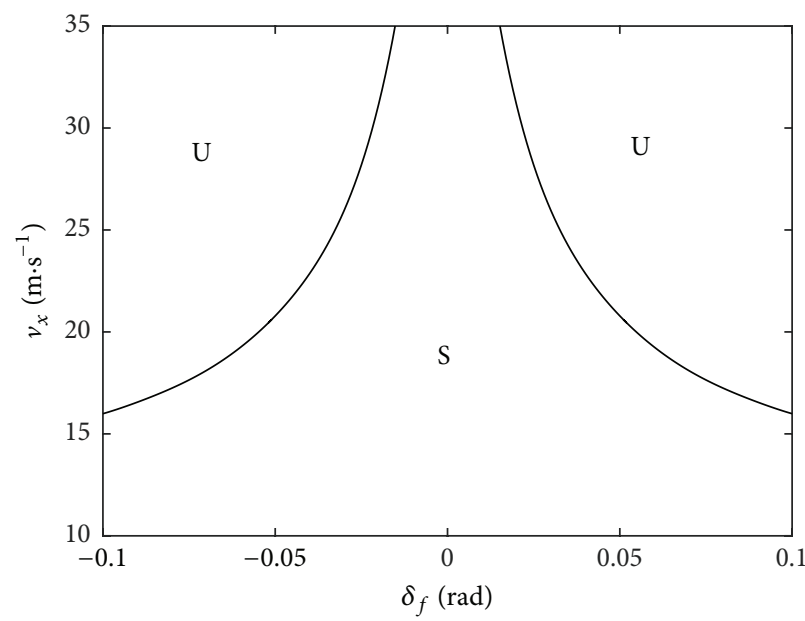

(a) $K=0$

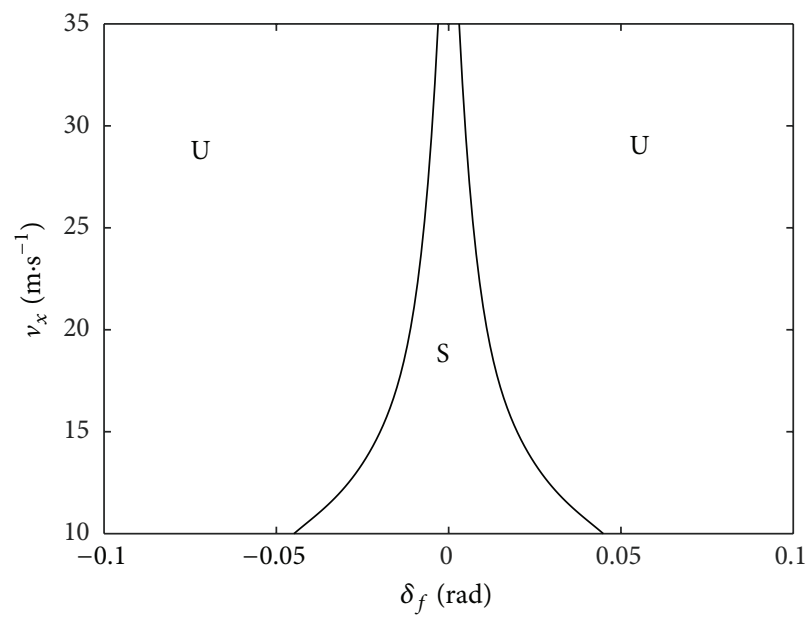

(c) $K=0$

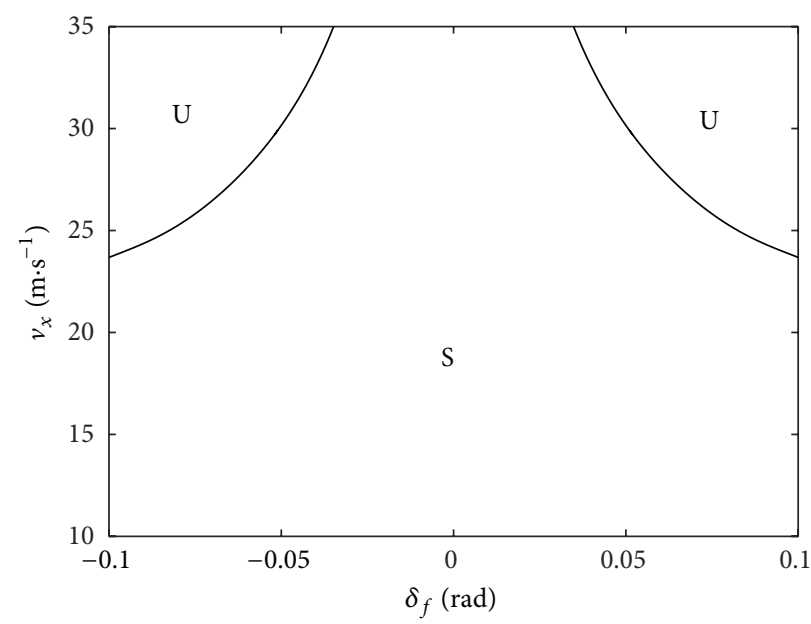

(b) $K=0.5$

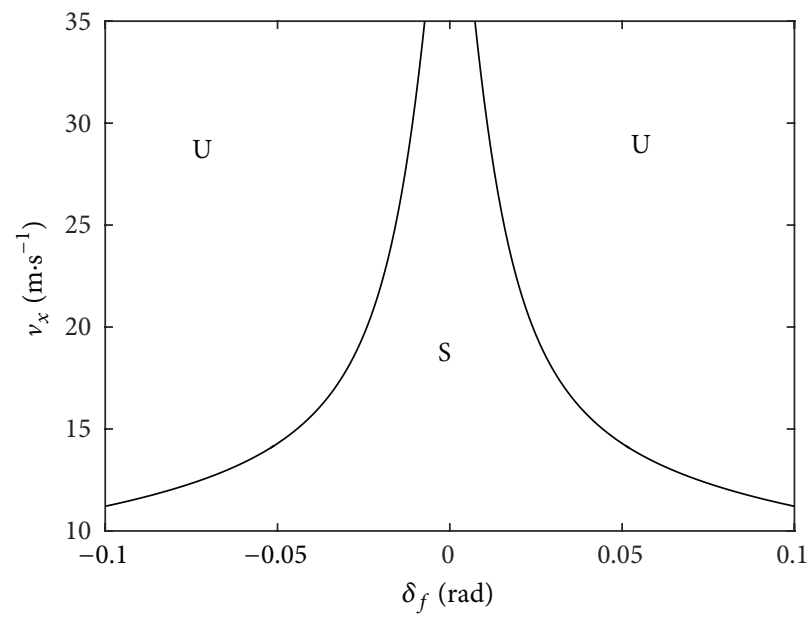

(d) $K=0.5$

FIgURE 8: The stable and unstable area of $v_{x}-\delta_{f}$. (a) and (b) High-mu friction; (c) and (d) low-mu friction.

boundary. Figure 9(b) shows that if only the rear wheels brake, the stable area is almost gone. For front wheels braking with low friction shown in Figure 10, the safe range of steering angle reduces very quickly as velocity $v_{x}$ increases. And any slight steering maneuver is not allowed when $v_{x}$ is up to a critical level such as $22 \mathrm{~m} / \mathrm{s}$ in Figure 10(a) and $28 \mathrm{~m} / \mathrm{s}$ in Figure 10(b). Comparing Figures 11 with 9, we observe that the four-wheel braking mode is not a good way to enlarge the stable area.

Generally, the application of 4WS system and front wheels braking improves vehicle motion stability. However, it is worth pointing out that

(1) Four-wheel braking should take the place of front wheels braking at high velocity on low-mu friction, to keep a certain steering capability (as seen in Figures 10(b) and 11(b));

(2) 4WS system is not useful at high velocity because it cannot widen the safe range for steering angle.
4.2. The Stable Area of Operating Conditions $s_{f}-s_{r}$. Tire slip ratio is a key factor for the stability of vehicle motion. The stability boundary is captured when vehicle is cornering with different slip ratio for front/rear wheel. We investigate how the variation of $s_{f}-s_{r}$ affects the vehicle motion stability for different circumstances. As shown in Figures 12-15, the influences of velocity, steering angle, friction surface, and steering system on the stable area are discussed, respectively. Corresponding to the above result, the stable area is reduced with $v_{x}$ or $\delta_{f}$ increasing. High-mu road surface and 4WS system are good for vehicle stability compared with low-mu and $2 \mathrm{WS}$, respectively.

The boundary indicates that large slip ratio of rear wheel is not allowed unless the braking/driving force of front wheel is strong enough during four-wheels braking/accelerating. As shown in Figure 12, the vehicle motion is always stable if $s_{r}$ is less than 0.25 when $v_{x}=15 \mathrm{~m} / \mathrm{s}$ or 0.1 when $v_{x}=20 \mathrm{~m} / \mathrm{s}$. Comparing Figures 14(a) with 14(b), we find that two unstable areas move closer and finally intersect at the origin of $s_{f}$ $s_{r}$ with strengthening trend of instability. And according to 


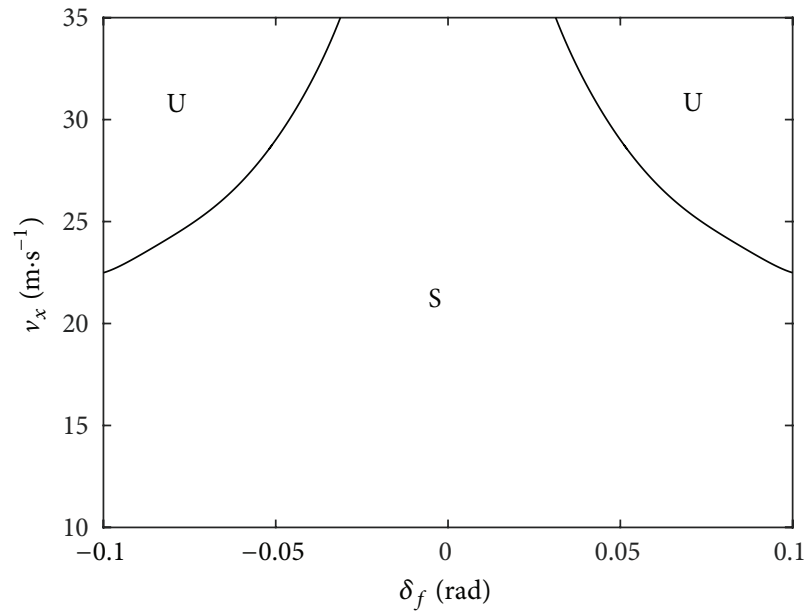

(a) $s_{f}=-0.2, s_{r}=0$

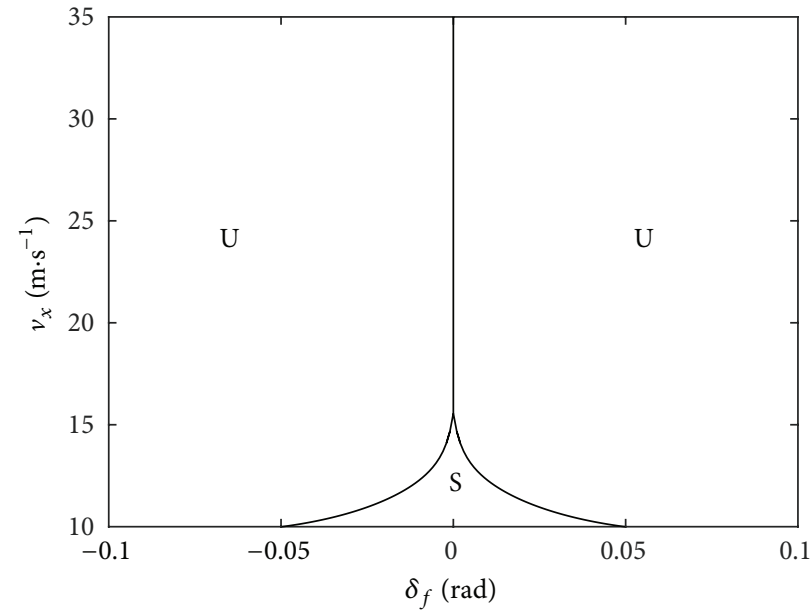

(b) $s_{f}=0, s_{r}=-0.2$

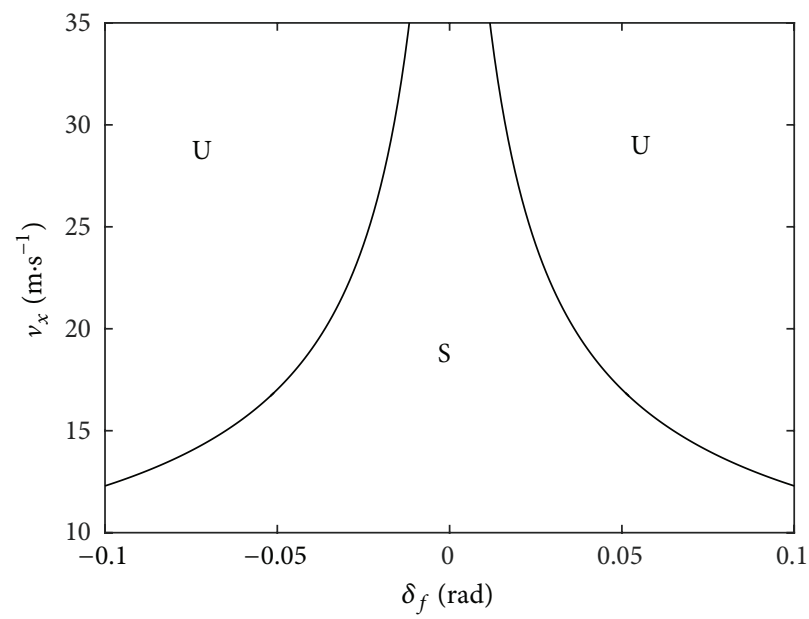

(c) $s_{f}=-0.2, s_{r}=-0.2$

Figure 9: The stable and unstable area of $v_{x}-\delta_{f}$. (a), (b), and (c) 2WS vehicles on high-mu road surface.

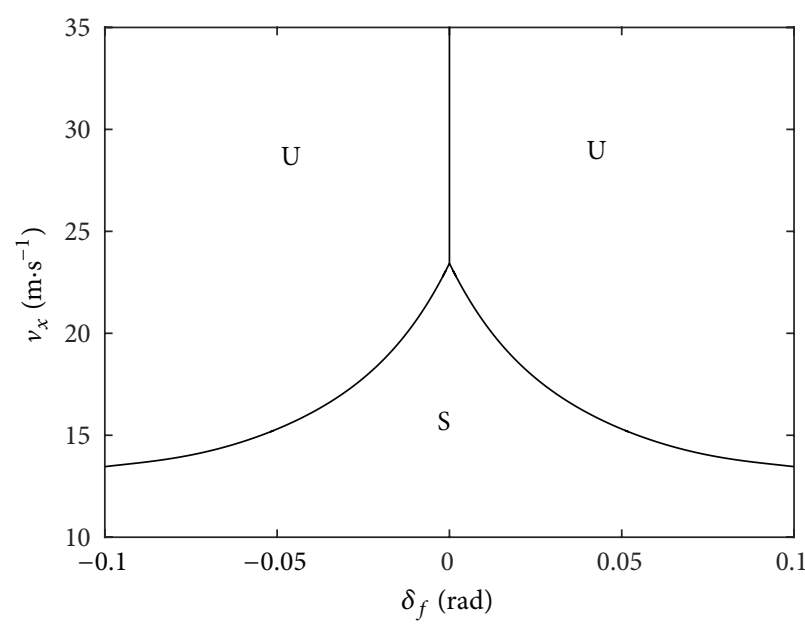

(a) $K=0$

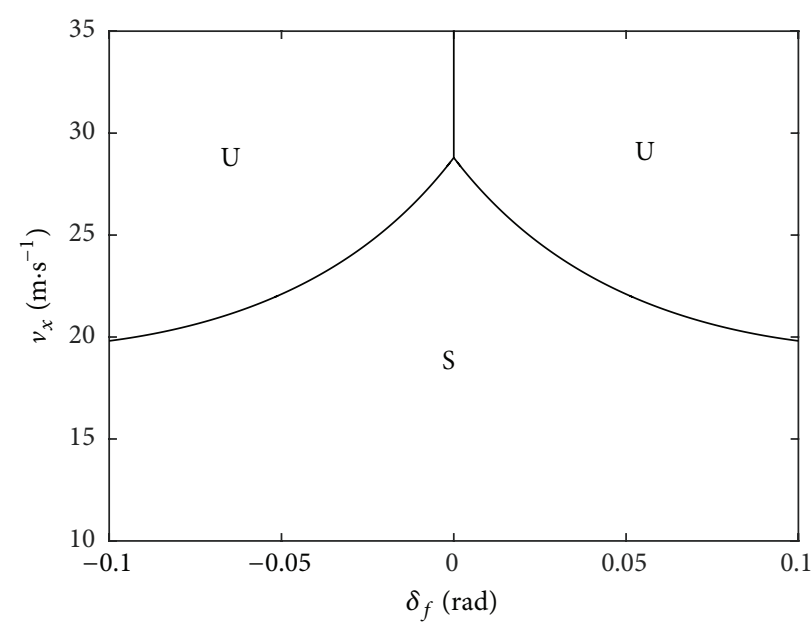

(b) $K=0.5$

Figure 10: The stable and unstable area of $v_{x}-\delta_{f}$. (a) and (b) $s_{f}=-0.2$ and $s_{r}=0$ on low-mu road surface. 


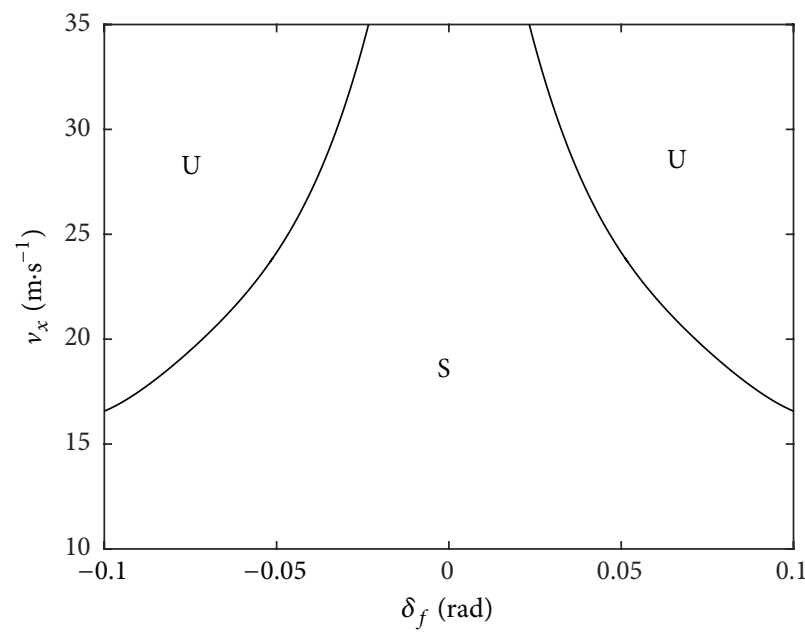

(a) High-mu friction

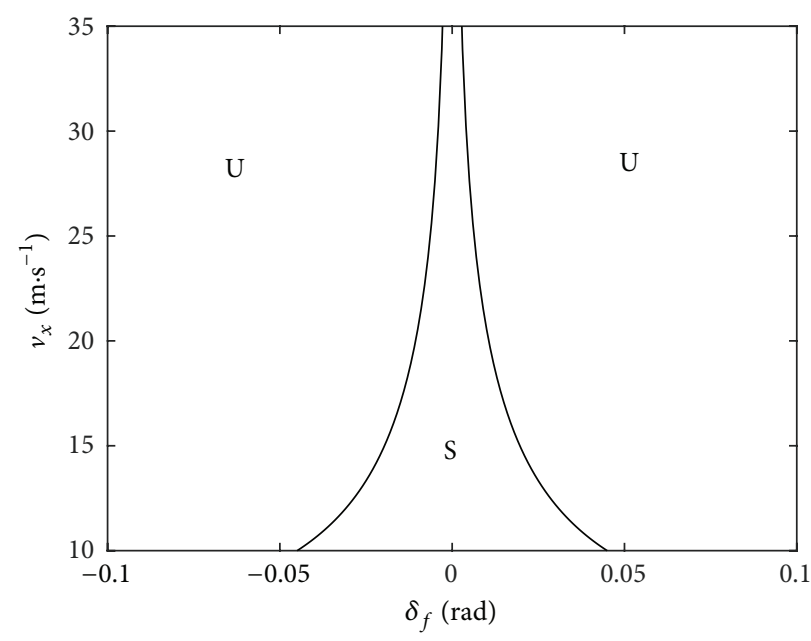

(b) Low-mu friction

FIGURE 11: The stable and unstable area of $v_{x}-\delta_{f}$. (a) and (b) $s_{f}=-0.2$ and $s_{r}=-0.2$ for 4 WS vehicles.

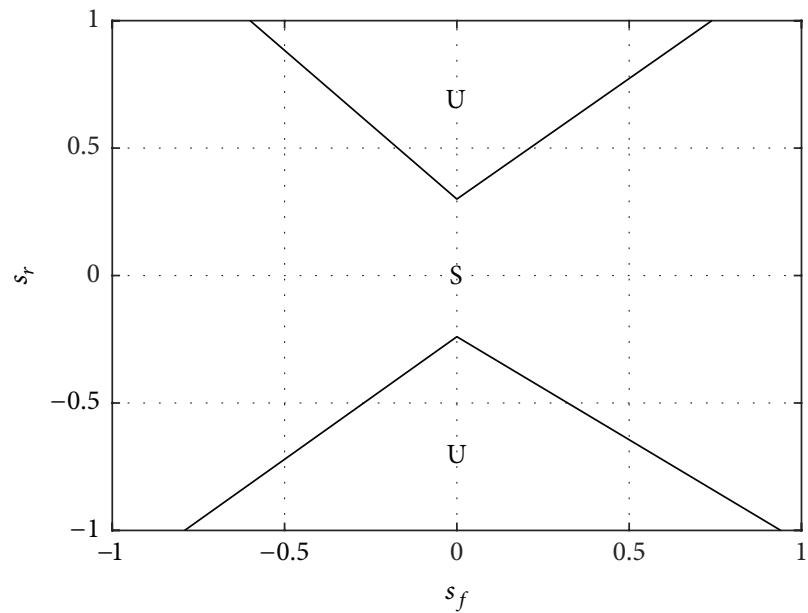

(a) $v_{x}=15 \mathrm{~m} / \mathrm{s}$

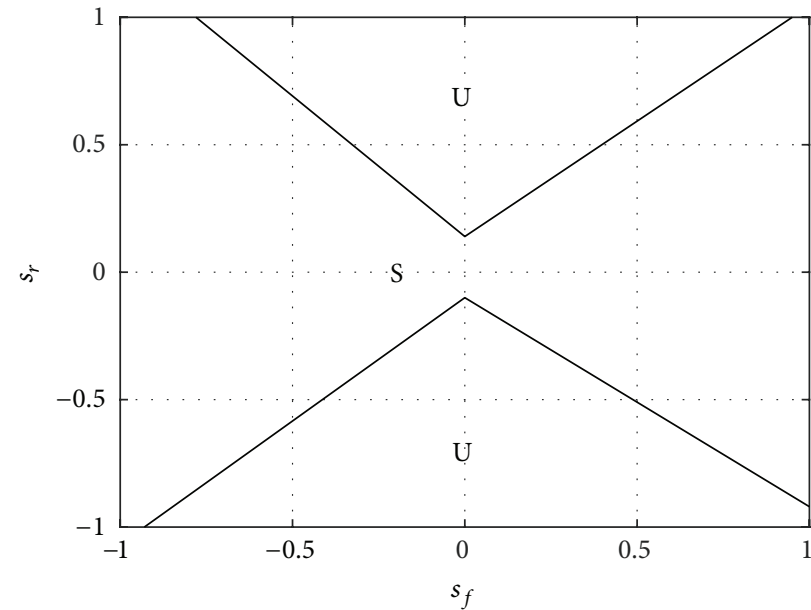

(b) $v_{x}=20 \mathrm{~m} / \mathrm{s}$

FIGURE 12: The stable and unstable area of $s_{f}-s_{r}$. (a) and (b) $\delta_{f}=0.01 \mathrm{rad}$ and $K=0.5$ for $4 \mathrm{WS}$ vehicles on high friction road.

the shape of these figures, weakening the rear braking/driving force or reinforcing the front one is an effective way to lead vehicle from instability to stability.

\section{Conclusion}

We present a coupled longitudinal/lateral stability analysis for vehicle planar motion. Under various operating conditions, the characteristic of vehicle stability is investigated by the phase portrait method and the stability boundaries are captured based on the eigenvalues of the Jacobian matrix at each equilibrium point. It is found by this paper that

(1) As velocity or steering angle increases, the stable equilibrium point moves towards the unstable saddle point in the phase plane; this results in the reduction of the stable region.
(2) High-mu road surface and 4WS are favorable conditions for vehicle stability, but the effect of 4WS is fairly limited for high speed vehicle.

(3) Compared with rear wheels braking or four-wheel braking, front wheels braking is more helpful to enlarge the stable area except on low friction road with high speed.

(4) If four-wheel braking or accelerating is necessary during vehicle corning, the slip ratio of front wheel should be ensured big enough with respect to the rear one.

The presented result provides a guide to handle the vehicle maneuvers safely. As future work, we intend to download the online MAP of vehicle stability boundary in ECU to keep the vehicle within the stable region. 


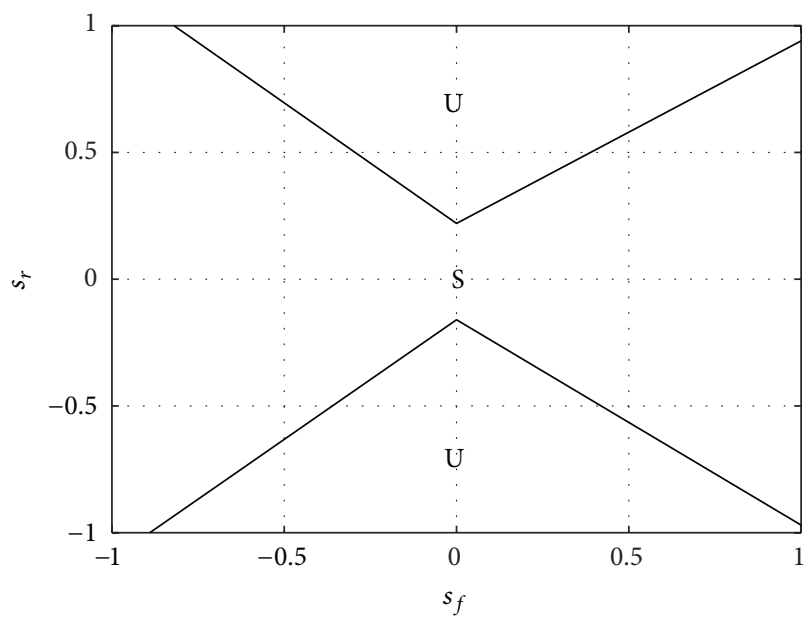

(a) $\delta_{f}=0.01 \mathrm{rad}$

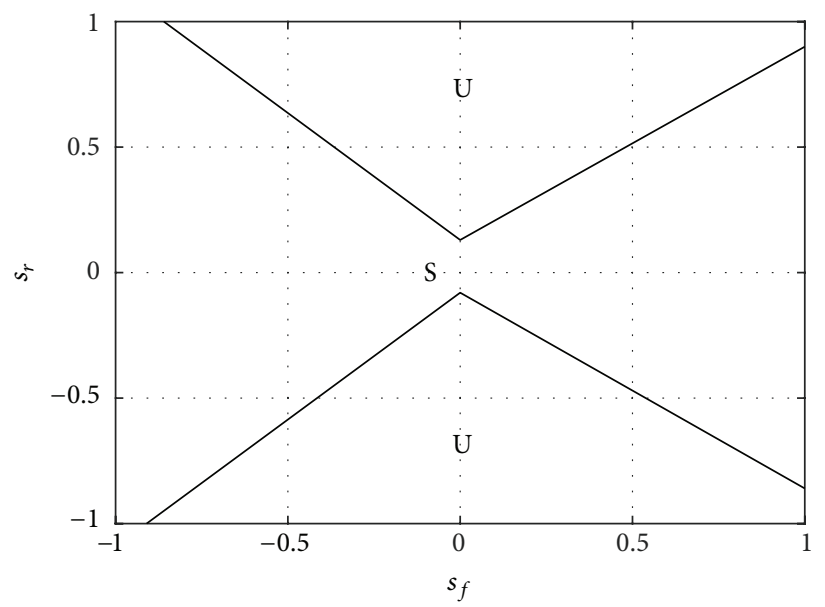

(b) $\delta_{f}=0.02 \mathrm{rad}$

FIGURE 13: The stable and unstable area of $s_{f}-s_{r}$. (a) and (b) $v_{x}=15 \mathrm{~m} / \mathrm{s}$ for 2 WS vehicles on high friction road.

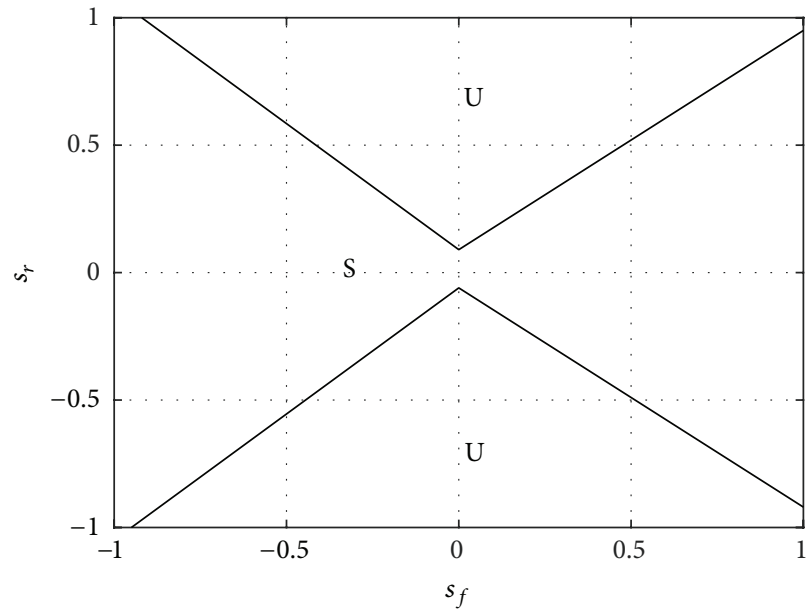

(a) High-mu friction

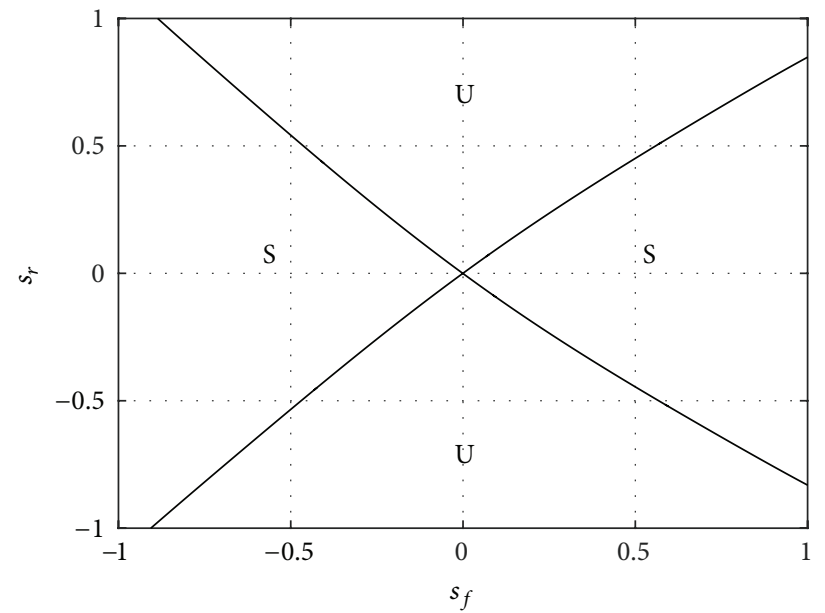

(b) Low-mu friction

FIGURE 14: The stable and unstable area of $s_{f}-s_{r}$. (a) and (b) $\delta_{f}=0.01 \mathrm{rad}$ and $v_{x}=20 \mathrm{~m} / \mathrm{s}$ for $2 \mathrm{WS}$ vehicles.

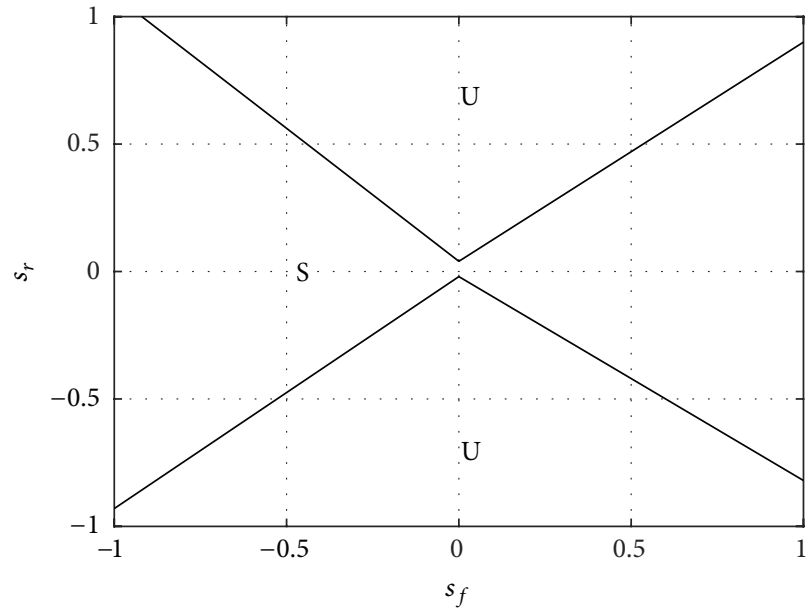

(a) $K=0$

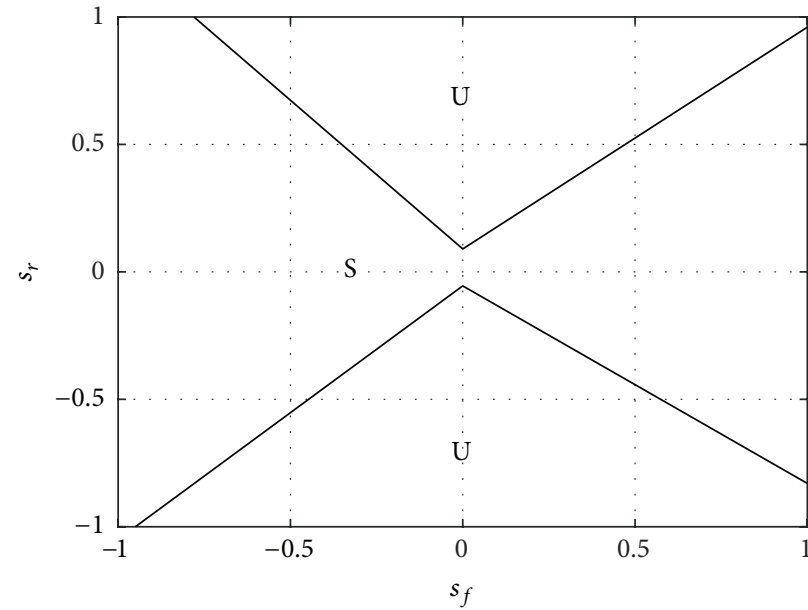

(b) $K=0.5$

FIGURE 15: The stable and unstable area of $s_{f}-s_{r}$. (a) and (b) $\delta_{f}=0.02 \mathrm{rad}$ and $v_{x}=20 \mathrm{~m} / \mathrm{s}$ on high friction road. 


\section{Appendix}

Consider

$$
\begin{aligned}
& \frac{\partial\left(\zeta_{f, r} P_{f, r}\right)}{\partial(\sigma, \omega)}=\frac{\partial \zeta_{f, r}}{\partial(\sigma, \omega)}+\frac{\partial P_{f, r}}{\partial(\sigma, \omega)}, \\
& \frac{\partial\left(\zeta_{f, r} Q_{f, r}\right)}{\partial(\sigma, \omega)}=\frac{\partial \zeta_{f, r}}{\partial(\sigma, \omega)}+\frac{\partial Q_{f, r}}{\partial(\sigma, \omega)},
\end{aligned}
$$

where

$$
\begin{aligned}
\frac{\partial \zeta_{f}}{\partial(\sigma, \omega)}= & -\frac{\left(\partial P_{f} / \partial(\sigma, \omega)-\partial P_{r} / \partial(\sigma, \omega)\right)\left(l_{r}-P_{r}\right)}{\left(L+P_{f}-P_{r}\right)^{2}} \\
& -\frac{\partial P_{r} / \partial(\sigma, \omega)}{L+P_{f}-P_{r}}, \\
\frac{\partial \zeta_{r}}{\partial(\sigma, \omega)}= & -\frac{\left(\partial P_{f} / \partial(\sigma, \omega)-\partial P_{r} / \partial(\sigma, \omega)\right)\left(l_{f}+P_{f}\right)}{\left(L+P_{f}-P_{r}\right)^{2}} \\
& +\frac{\partial P_{r} / \partial(\sigma, \omega)}{L+P_{f}-P_{r}}
\end{aligned}
$$

and, respectively,

$$
\begin{aligned}
& \frac{\partial P_{f, r}}{\partial(\sigma, \omega)}=h\left(\frac{\partial \mu_{f, r}}{\partial(\sigma, \omega)} \cos \delta_{f, r}-\frac{\partial \eta_{f, r}}{\partial(\sigma, \omega)} \sin \delta_{f, r}\right) \\
& \frac{\partial Q_{f, r}}{\partial(\sigma, \omega)}=\frac{\partial \mu_{f, r}}{\partial(\sigma, \omega)} \sin \delta_{f, r}+\frac{\partial \eta_{f, r}}{\partial(\sigma, \omega)} \cos \delta_{f, r} \cdot \\
& \text { If } \alpha_{f, r} \geq 0, \text { then } \\
& \frac{\partial \mu_{f, r}}{\partial(\sigma, \omega)}=k_{1}\left[\operatorname{sgn}\left(s_{f, r}\right) k_{x 1}\left(1-e^{\operatorname{sgn}\left(s_{f, r}\right) k_{x 2} \cdot s_{f, r}}\right)\right. \\
& \left.+k_{x 3} \cdot s_{f, r}\right] \frac{\partial \tan \alpha_{f, r}}{\partial(\sigma, \omega)}, \\
& \frac{\partial \eta_{f, r}}{\partial(\sigma, \omega)}=-\left(\frac{k_{2}\left|s_{f, r}\right|+1}{k_{3}\left|s_{f, r}\right|+1}\right)\left(-k_{y 1} k_{y 2} e^{k_{y 2} \cdot \tan \left(\alpha_{f, r}\right)}\right. \\
& \left.\quad+k_{y 3}\right) \frac{\partial \tan \alpha_{f, r}}{\partial(\sigma, \omega)} ;
\end{aligned}
$$

else

$$
\begin{aligned}
& \frac{\partial \mu_{f, r}}{\partial(\sigma, \omega)}=-k_{1}\left[\operatorname{sgn}\left(s_{f, r}\right) k_{x 1}\left(1-e^{\operatorname{sgn}\left(s_{f, r}\right) k_{x 2} \cdot s_{f, r}}\right)\right. \\
& \left.+k_{x 3} \cdot s_{f, r}\right] \frac{\partial \tan \alpha_{f, r}}{\partial(\sigma, \omega)}, \\
& \frac{\partial \eta_{f, r}}{\partial(\sigma, \omega)}=-\left(\frac{k_{2}\left|s_{f, r}\right|+1}{k_{3}\left|s_{f, r}\right|+1}\right)\left(-k_{y 1} k_{y 2} e^{-k_{y 2} \cdot \tan \left(\alpha_{f, r}\right)}\right. \\
& \left.\quad+k_{y 3}\right) \frac{\partial \tan \alpha_{f, r}}{\partial(\sigma, \omega)}
\end{aligned}
$$

finally

$$
\begin{aligned}
& \frac{\partial \tan \alpha_{f}}{\partial \sigma}=\frac{\sec ^{2}\left[\arctan \left(\sigma+\left(l_{f} / v_{x}\right) \omega\right)-\delta_{f}\right]}{1+\left(\sigma+\left(l_{f} / v_{x}\right) \omega\right)^{2}}, \\
& \frac{\partial \tan \alpha_{f}}{\partial \omega}=\frac{\partial \alpha_{f}}{\partial \sigma} \cdot \frac{l_{f}}{v_{x}}, \\
& \frac{\partial \tan \alpha_{r}}{\partial \sigma}=\frac{\sec ^{2}\left[\arctan \left(\sigma-\left(l_{r} / v_{x}\right) \omega\right)-\delta_{r}\right]}{1+\left(\sigma-\left(l_{r} / v_{x}\right) \omega\right)^{2}}, \\
& \frac{\partial \tan \alpha_{r}}{\partial \omega}=-\frac{\partial \alpha_{r}}{\partial \sigma} \cdot \frac{l_{r}}{v_{x}} .
\end{aligned}
$$

\section{Competing Interests}

The authors declared that there is no conflict of interests regarding the publication of this paper.

\section{Acknowledgments}

This work was supported in part by the National Natural Science Foundation of the People's Republic of China (Grant no. 51505354).

\section{References}

[1] J. Lu, D. Filev, K. Prakah-Asante, F. Tseng, and I. V. Kolmanovsky, "From vehicle stability control to intelligent personal minder: real-time vehicle handling limit warning and driver style characterization," in Proceedings of the IEEE on Workshop Computational Intelligence in Vehicles and Vehicular Systems (CIVVS '09), pp. 43-50, Nashville, Tenn, USA, March-April 2009.

[2] Y.-J. Liu and S. Tong, "Adaptive fuzzy control for a class of unknown nonlinear dynamical systems," Fuzzy Sets and Systems, vol. 263, pp. 49-70, 2015.

[3] F. Wang, Z. Liu, Y. Zhang, X. Chen, and C. L. P. Chen, "Adaptive fuzzy dynamic surface control for a class of nonlinear systems with fuzzy dead zone and dynamic uncertainties," Nonlinear Dynamics, vol. 79, no. 3, pp. 1693-1709, 2014.

[4] K. Zhao, J. Wang, X. Xu, and Z. Huang, "An approximate dynamic programming approach for path following control of an autonomous vehicle," in Proceedings of the 11th World Congress on Intelligent Control and Automation (WCICA '14), pp. 1998-2004, Shenyang, China, July 2014.

[5] Y.-J. Liu, Y. Gao, S. Tong, and Y. Li, "Fuzzy approximation-based adaptive backstepping optimal control for a class of nonlinear discrete-time systems with dead-zone," IEEE Transactions on Fuzzy Systems, vol. 24, no. 1, pp. 16-28, 2016.

[6] G. Lai, Z. Liu, Y. Zhang, and C. L. Philip Chen, "Adaptive fuzzy tracking control of nonlinear systems with asymmetric actuator backlash based on a new smooth inverse," IEEE Transactions on Cybernetics, vol. 46, no. 6, pp. 1250-1262, 2016.

[7] R. Rizvi, S. Kalra, C. Gosalia, and S. Rahnamayan, "Fuzzy adaptive cruise control system with speed sign detection capability," in Proceedings of the IEEE International Conference on Fuzzy Systems (FUZZ-IEEE '14), pp. 968-976, IEEE, Beijing, China, July 2014. 
[8] Z. Li, J. Deng, R. Lu, Y. Xu, J. Bai, and C. Su, “Trajectory-tracking control of mobile robot systems incorporating neural-dynamic optimized model predictive approach," IEEE Transactions on Systems, Man, and Cybernetics: Systems, vol. 46, no. 6, pp. 740749, 2016.

[9] D. Zhang, K. Li, and J. Wang, "A curving ACC system with coordination control of longitudinal car-following and lateral stability," Vehicle System Dynamics, vol. 50, no. 7, pp. 1085-1102, 2012.

[10] J. Wang, J. Steiber, and B. Surampudi, "Autonomous ground vehicle control system for high-speed and safe operation," in Proceedings of the American Control Conference (ACC '08), pp. 218-223, Seattle, Wash, USA, June 2008.

[11] P. Falcone, H. Eric Tseng, F. Borrelli, J. Asgari, and D. Hrovat, "MPC-based yaw and lateral stabilisation via active front steering and braking," Vehicle System Dynamics, vol. 46, no. 1, pp. 611-628, 2008.

[12] J.-S. Jo, S.-H. You, J. Y. Joeng, K. I. Lee, and K. Yi, "Vehicle stability control system for enhancing steerabilty, lateral stability, and roll stability, International Journal of Automotive Technology, vol. 9, no. 5, pp. 571-576, 2008.

[13] D.-F. Li and F. Yu, "Integrated vehicle dynamics controller design based on optimum tire force distribution," Journal of Shanghai Jiaotong University, vol. 42, no. 6, pp. 887-891, 2008.

[14] W. Cho, J. Choi, C. Kim, S. Choi, and K. Yi, "Unified chassis control for the improvement of agility, maneuverability, and lateral stability," IEEE Transactions on Vehicular Technology, vol. 61, no. 3, pp. 1008-1020, 2012.

[15] S. Inagaki, I. Kshiro, and M. Yamamoto, "Analysis of vehicle stability in critical cornering using phase plane method," SAE Paper 943841, 1994.

[16] E. Ono, S. Hosoe, H. D. Tuan, and S. Doi, "Bifurcation in vehicle dynamics and robust front wheel steering control," IEEE Transactions on Control Systems Technology, vol. 6, no. 3, pp. 412-420, 1998.

[17] B. J. Olson, S. W. Shaw, and G. Stépán, "Stability and bifurcation of longitudinal vehicle braking," Nonlinear Dynamics, vol. 40, no. 4, pp. 339-365, 2005.

[18] L. Dai and Q. Han, "Stability and Hopf bifurcation of a nonlinear model for a four-wheel-steering vehicle system," Communications in Nonlinear Science and Numerical Simulation, vol. 9, no. 3, pp. 331-341, 2004.

[19] S. Shen, J. Wang, P. Shi, and G. Premier, "Nonlinear dynamics and stability analysis of vehicle plane motions," Vehicle System Dynamics, vol. 45, no. 1, pp. 15-35, 2007.

[20] D.-C. Liaw, H.-H. Chiang, and T.-T. Lee, "Elucidating vehicle lateral dynamics using a bifurcation analysis," IEEE Transactions on Intelligent Transportation Systems, vol. 8, no. 2, pp. 195207, 2007.

[21] J. Li, Y. Zhang, J. Yi, and Z. Liu, "Understanding agile-maneuver driving strategies using coupled longitudinal/lateral vehicle dynamics," in Proceedings of the ASME 4th Annual Dynamic Systems and Control Conference, Arlington, Va, USA, OctoberNovember 2011.

[22] J. Yi, J. Li, J. Lu, and Z. Liu, "On the stability and agility of aggressive vehicle maneuvers: a pendulum-turn maneuver example," IEEE Transactions on Control Systems Technology, vol. 20, no. 3, pp. 663-676, 2012.

[23] H. B. Pacejka, Tire and Vehicle Dynamics, ButterworthHeinemann, Oxford, UK, 2002.
[24] Z. Qi, S. Taheri, B. Wang, and H. Yu, "Estimation of the tyreroad maximum friction coefficient and slip slope based on a novel tyre model," Vehicle System Dynamics, vol. 53, no. 4, pp. 506-525, 2015.

[25] W. Liang, J. Medanic, and R. Ruhl, "Analytical dynamic tire model," Vehicle System Dynamics, vol. 46, no. 3, pp. 197-227, 2008.

[26] M. Burckhardt, Fahrwerktechnik, Radschlupf-Regelsysteme, Vogel Buchverl, Würzburg, Germany, 1993. 


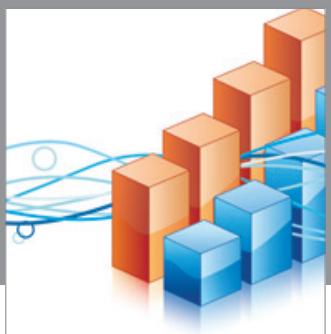

Advances in

Operations Research

vatem alat4

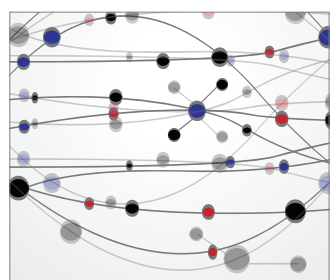

\section{The Scientific} World Journal
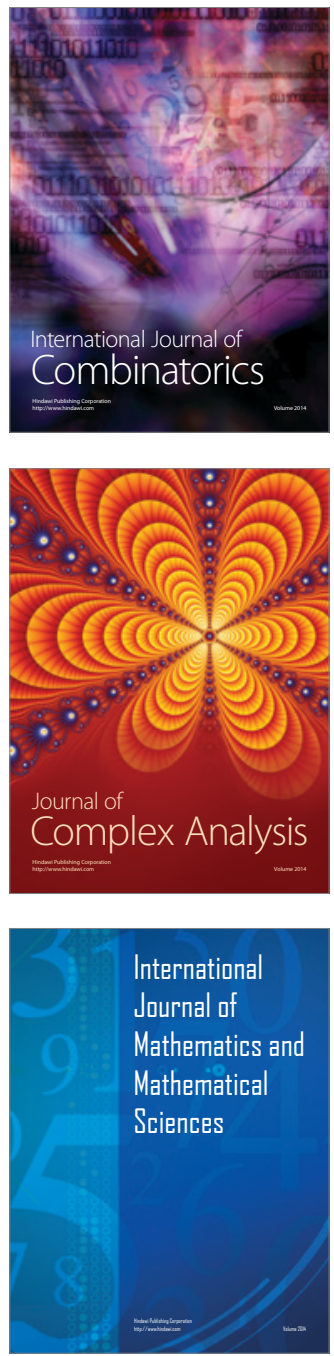
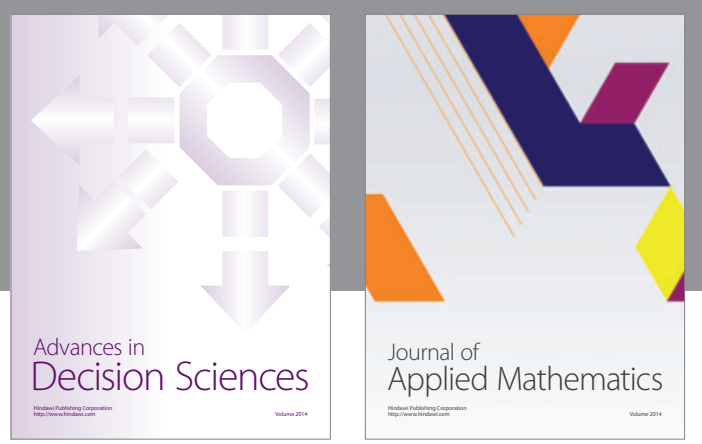

Algebra

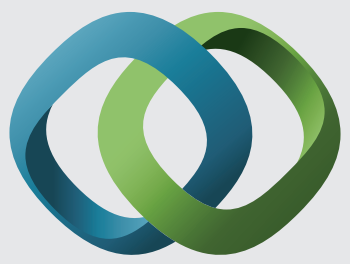

\section{Hindawi}

Submit your manuscripts at

http://www.hindawi.com
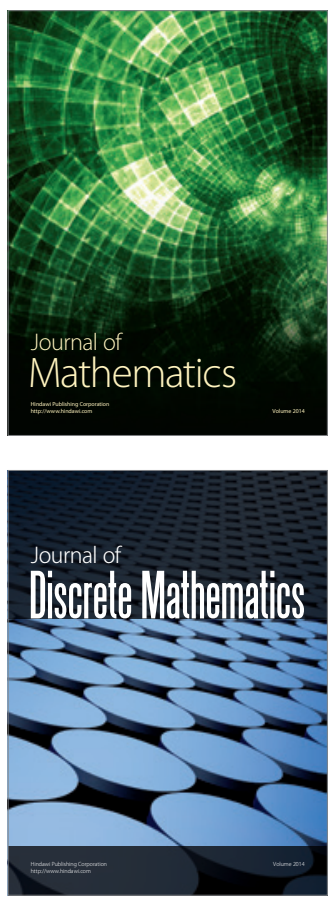

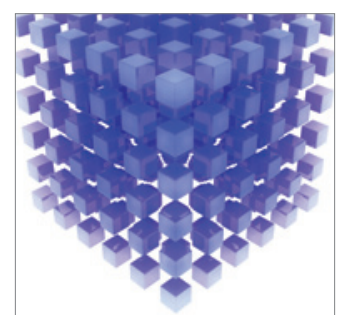

Mathematical Problems in Engineering
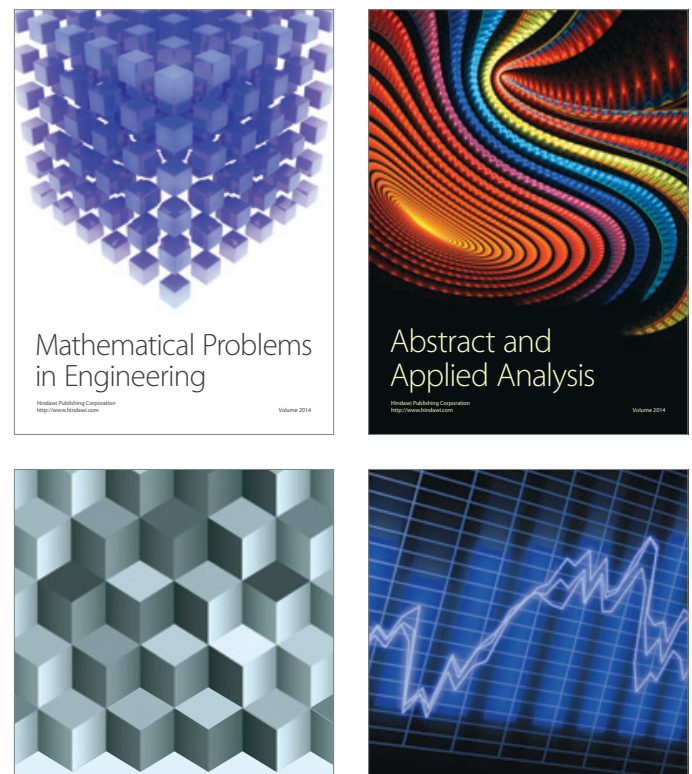

Journal of

Function Spaces

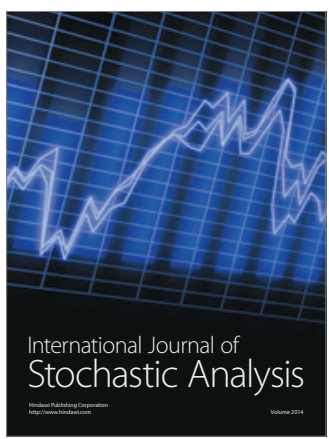

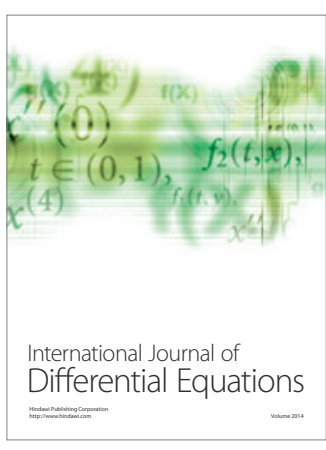
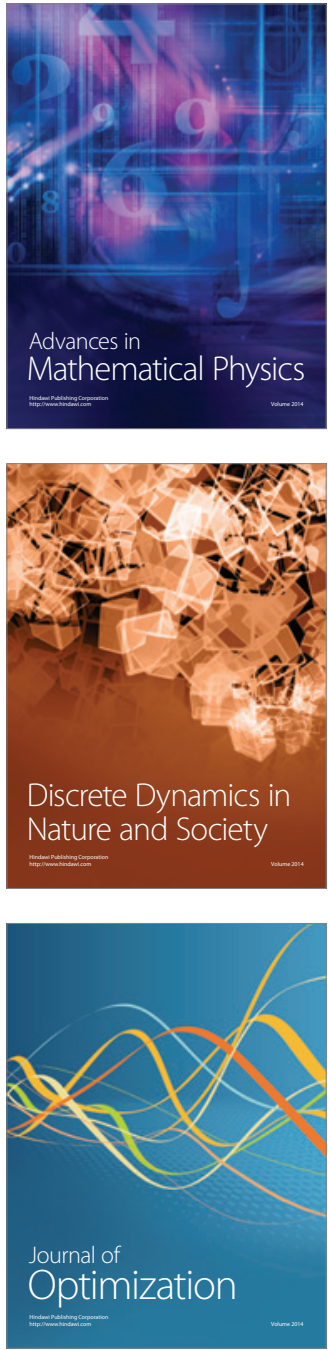